\title{
XXII.
}

\section{SOPRA ALCUNE QUESTIONI \\ DI INVERSIONE DI INTEGRALI DEFINITI}

\author{
"Annali di Matematica Pura ed Applicata ", \\ ser. $2^{\text {a }}$, vol. XXV, I897, pp. I39-1 78 .
}

In una serie di Note ${ }^{(x)}$ che ho presentato quest'anno alle. Accademie di Torino e dei Lincei, ho esposto un metodo con cui può risolversi il problema della inversione degli integrali definiti.

Mi permetto ora di portare un contributo alle dette ricerche con questa Memoria la quale consta di tre parti. Neila prima dò un brevissimo cenno di studi fatti in passato sulla questione ed esamino i principii sui quali è fondato il mio metodo. Nella seconda applico il metodo stesso al caso della inversione quando ambedue i limiti sono variabili. Nella terza infine considero alcune questioni particolari, mostrando i risultati che si ottengono allorché si eseguiscono quelle quadrature mediante le quali il detto procedimento risolve i problemi di inversione.

ART. I.

I. Il seguire le vicende del problema della inversione degli integrali definiti potrebbe dar luogo ad una pagina istruttiva ed interessante di storia dell'analisi, giacché una tale ricerca, intimamente legata alla integrazione delle equazioni differenziali, agli sviluppi in serie e ad una classe estesa di questioni fisico-matematiche, si è imposta di frequente all'attenzione dei geometri. Senza accingerci, per la sua difficoltà, a tale impresa, accenneremo solo ad alcuni dei principali lavori a nostra conoscenza sull'argomento, chiedendo fin da principio venia delle involontarie omissioni.

2. Il maraviglioso risultato stabilito da ABEL nel I $823^{(*)}$ colle sue due formule reciproche:

$$
\psi(a)=\int_{0}^{a} \frac{d s}{(a-x)^{n}} \quad, \quad s=\frac{\operatorname{sen} n \pi}{\pi} x^{n} \int_{0}^{1} \frac{\psi(x t) d t}{(1-t)^{\mathrm{I}-n}}, \quad(n<\mathrm{I}),
$$

è così noto che ci basta solo di ricordarlo.

(I) Sulla inversione degli integrali definiti. Note I, II, III, IV "Atti della R. Accademia delle Scienze di Torino", 12 gennaio, 26 gennaio, 8 marzo, 26 aprile 1896 [in questo vol.: XVIII, pp. 216-254]. Sulla inversione degli integrali definiti. "Rend. della R. Accademia dei Lincei ", $1^{\circ}$ marzo 1896 [ibidem: XIX, pp. 255-262]. Sulla inversione degli integrali multipli, 26 aprile 1896 [ibidem: XX, pp. 263-278].

$\left({ }^{*}\right)$ Solution de quelques problèmes etc. "Oeuvres", nouv. ed., t. I, pp. II-27. [N. d. R.]. 
Osserviamo soltanto che l'A. conclude il § I della detta Memoria colle seguenti parole:

"Je remarquerai enfin que de la même manière qu'en partant de l'équation:

$$
\psi(a)=\int_{x=0}^{x=a} \frac{d s}{(a-x)^{n}},
$$

j'ai trouvé $s$, de même en partant de l'équation:

$$
\psi(a)=\int \varphi(x a) f(x) d x
$$

j'ai trouvé la fonction $\varphi, \psi$ et $f$ étant des fonctions données et l'intégrale étant prise entre des limites quelconques; mais la solution de ce problème est trop longue pour être donnée ici ».

Questo scriveva ABEL nel I823: tre anni dopo ripubblicava nel "Giornale di Crelle " sotto il titolo: Résolution d'un problème de mécanique(*) la stessa formula di inversione della equazione (I), ma non dava alcun cenno di aver risolto la questione generale di invertire la (2). Né il problema stesso riappare in alcuna altra Memoria di ABEL. Ci mancano quindi i dati necessari per decidere la questione se ABEL fosse effettivamente giunto a sciogliere questo problema o invece non avesse creduto per un momento alla possibilità di risolverlo seguendo una certa via, senza che le sue ulteriori ricerche abbiano confermato questa credenza.

Quest'ultima ipotesi è resa più probabile dal fatto che HoLmboE, che doveva conoscere le idee definitive di ABEL su questo soggetto, ha creduto di sopprimere il paragrafo della Memoria che contiene il solo passo delle opere in cui ABEL afferma di aver risoluto questo problema.

Certo è che nessuno dei molti matematici che in appresso si occuparono della questione, giunse, che io sappia, ad ottenerne la soluzione.

Nell'Art. II mostreremo come essa possa ricavarsi col metodo ivi esposto che è fondato sopra principii che non hanno alcun rapporto col metodo che seguiva ABEL.

3. Non erano ancora trascorsi tre anni dalla morte di ABEL che, nel I832, LIOUVILLE stabiliva alcune formule d'inversione che in ultima analisi possono ricavarsi da quella d'ABEL e le applicava a numerose ed importanti questioni di varia natura, le quali rendono sommariamente interessante la lettura della sua Memoria. Il LIOUviLLE era ignaro delle ricerche d'ABEL, giacché applicando la sua formula alla estensione del problema della tautocrona dice, dopo enunciato il problema: "Les recherches des divers géomètres sur la tautochrone ne s'étendraient nullement à la question plus difficile qui vient d'être énoncée ", mentre il problema stesso era stato risolto da ABEL nove anni prima.

(*) "Crelle", Bd. I (1826); "Oeuvres" nouv. ed., t. I, pp. 97-10I. [N. d. R.]. 
Liouville però, più che alla questione funzionale mirava alla creazione di un nuovo calcoio: quello delle derivate d'indice fratto. Ma se questo suo concepimento contribuisce a dare forma elegantissima ai risultati, esso per altro lo costringe rispetto alla questione funzionale nei limiti della formula d'ABEL.

Liouville era conscio di tutta la importanza delle questioni funzionali nell'analisi delle equazioni a derivate parziali e specialmente nella fisica matematica. Dopo avere esaminato una estesa classe di questioni fisico meccaniche, egli dice: "tous ces problèmes sur les forces attractives se rapportent à une branche nouvelle des théories mécaniques dans laquelle on a pour but d'obtenir les forces élémentaires connaissant la loi que suivent dans une série régulière de cas donnés les résultantes de ces forces élémentaires. L'objet qu'on se propose dans cette partie de la science est de remonter des effets aux causes ").

Ora LIOUVILLE riteneva necessario di dover ricorrere ad " un nuovo calcolo " per risolvere le questioni suddette; ma esse posson farsi rientrare in quello ordinario con altrettanta facilità.

Nondimeno la eleganza delle formule di LiouviLle ha invoglia to vari autori a valersi delle sue derivate d'ordine fratto, e i loro risultati sono facilmente traducibili nell'ordinario linguaggio colle notazioni ordinarie.

4. Tanto ABEL quanto Liouville per stabilire le loro formule adoperavano il metodo degli sviluppi in serie. Esso però non è necessario, e, quel che è più importante di notare, non è rigoroso. Nel I 848 SCHLÖMILCH nei suoi analytische Studien riconobbe che la dimostrazione di ABEL giace sopra un fondamento inesatto e particolarizzando una formula generale relativa alla riduzione degli integrali multipli ottenne la formula di ABEL in maniera rigorosa.

Le dimostrazioni che più comunemente si dànno di essa sono però di natura diversa della precedente. Esse si basano sopra una formola di DIRICHLET. Questi fino dal I 835 nella sua Memoria: Sur les séries dont le terme général dépend de deux angles et qui servent à exprimer des fonctions arbitraires entre des limites données (*), aveva stabilito quel principio sulla inversione dell'ordine d'integrazione degli integrali doppii che è di tanta utilità e così frequentemente adoperato.

Esso si esprime mediante la formula:

$$
\int_{a}^{b} d x \int_{a}^{x} \varphi(x, y) d x=\int_{a}^{b} d y \int_{y}^{b} \varphi(x, y) d x,
$$

la quale, come dice il suo scopritore, " est très-facile de démontrer et qui devient tout à fait évidente, lorsqu'on l'envisage sous un point de vue géométrique ", ma che può ottenersi pur facilmente per via analitica, come ha mostrato WINCKLER nel I $869^{(2)}$.

$(*)$ "Crelle", Bd. 17 (1826); "Werke", Bd. I, pp. 283-306. [N. d. R.].

(2) La precedente formula di DiRICHLET può estendersi, sotto certe condizioni, al caso in cui $x, y$ siano variabili complesse. [Cfr. il § Io della prima nota precedentemente citata]. 
$\mathrm{Fu}$ JOACHIMSTHAL nel i 860 che per primo riconobbe, nel risolvere un problema meccanico proposto da JuLLIEN, come il suddetto principio di DiRICHLET possa impiegarsi utilmente per ottenere la formola d'inversione di ABEL.

Non starò ad esporre in qual modo il principio di DIRICHLET renda quasi intuitiva questa formula, giacché tale procedimento è ormai famigliare a tutti i cultori dell'analisi. Accennerò soltanto che il BELTRAMI, il DinI, il BETTI si valsero di esso nelle loro belle ricerche sulla teoria del potenziale. LETNIKOFF nel suo studio sulle derivate con indice fratto ne fa pure uso e così molti altri che non starò a citare.

Tuttavia i metodi escogitati per dimostrare la formula d'ABEL non si limitano ai precedenti. Ricorderò fra le altre dimostrazioni una molto elegante del Somoff che la espose nel i 868 nella sua Nota: Sur un problème de mécanique, nella quale egli estese anche il problema della tautocrona, proponendosi di trovare la curva descritta sopra una superficie qualunque da un punto sollecitato da una forza che ha un dato potenziale conoscendo il tempo in funzione del potenziale. L'Autore risolve questo problema più generale facendo sempre uso della stessa formula d'ABEL.

5. Le applicazioni di questa non sono ristrette alle sole ricerche fisicomeccaniche di cui abbiamo parlato, né a quelle solo analitiche di LiouviLLE. Una geniale idea emessa da SchlömILCH fu la fonte da cui scaturirono le applicazioni di essa agli sviluppi in serie, alle quali sono legate le profonde ricerche di BELTRAMI e di Dini di cui fra poco daremo un cenno.

Nel $\$ 7$ della sua Memoria: Ueber die Bessel'sche Funktion, ScHLömILcH si propone di sviluppare una funzione arbitraria di una variabile in una serie di funzioni di BEssel. Egli parte dallo sviluppo di FoURIER:

$$
\mathrm{F}(\lambda x)=\frac{1}{2} \mathrm{~A}_{0}+\mathrm{A}_{1} \cos 2 \lambda x+\mathrm{A}_{2} \cos 4 \lambda x+\cdots,
$$

in cui:

$$
\mathrm{A}_{n}=\frac{4}{\pi} \int_{0}^{\pi / 2} \mathrm{~F}(u) \cos 2 n u d u
$$

quindi moltiplicando per:

$$
\frac{2}{\pi} \frac{d x}{\sqrt{1-x^{2}}}
$$

ed integrando fra o ed I, giunge alla formula ${ }^{(3)}$ :

(3) Si osservi che Schlömilch fa uso della notazione di HANSEN anziché di quella di BESSEL, per cui si ha:

$$
J_{0}(\lambda)=\frac{2}{\pi} \int_{0}^{1} \frac{\cos 2 \lambda x}{\sqrt{1-x^{2}}} d x
$$

$\operatorname{ossia} \mathrm{J}_{\circ}(\lambda)=\mathrm{I}_{\circ}(2 \lambda)$ 


$$
\frac{2}{\pi} \int_{0}^{I} \frac{F(\lambda x)}{\sqrt{I-x^{2}}} d x=\frac{1}{2} A_{0}+A_{1} J_{0}(\lambda)+A_{2} J_{0}(2 \lambda)+A_{3} J_{0}(3 \lambda)+\cdots,
$$

che è valida per $\lambda$ compresa fra $\circ$ e $\frac{\mathrm{I}}{2} \pi$.

Ponendo:

$$
f(\lambda)=\frac{2}{\pi} \int_{0}^{I} \frac{F(\lambda x) d x}{\sqrt{1-x^{2}}}
$$

SCHLÖmILCH ha in tal modo lo sviluppo richiesto di $f(\lambda)$ per funzioni di BESSEL, onde in virtù delle (3) si potranno determinare i coeffiçienti dello sviluppo, purché dalla equazione precedente possa ricavarsi $\mathrm{F}$ in funzione di $f$. $\overrightarrow{\mathrm{E}}$ appunto questo problema d'inversione che SCHLÖMILCH risolve mediante la formula d'ABEL. In seguito l'Autore ottiene lo sviluppo analogo di una funzione per funzioni cilindriche d'ordine uno.

6. Nel i 880 il prof. BeLtRAMI ${ }^{*}$ ) ha preso nuovamente in esame la formula d'ABEL e la ha presentata sotto una forma atta a risolvere simultaneamente due questioni reciproche, l'una delle quali è quella precedentemente citata di SCHLÖmILCH, e l'altra una nuova questione.

Il BeLtRAmi osserva che il carattere essenziale della scoperta d'ABEL si può far consistere nella equivalenza delle due relazioni:

$$
\left\{\begin{array}{l}
\int_{0}^{\pi} \varphi(r \operatorname{sen} \theta) d \theta=\psi(r) \\
\int_{0}^{r} \varphi(r) d r=\frac{r}{\pi} \int_{0}^{\pi / 2} \psi(r \operatorname{sen} \theta) \operatorname{sen} \theta d \theta .
\end{array}\right.
$$

Tenendo ora conto delle due formule:

$$
\left\{\begin{array}{l}
\int_{0}^{\pi} \cos (x \operatorname{sen} \theta) d \theta=\pi I_{0}(x) . \\
\int_{0}^{\pi} \operatorname{sen}(x \operatorname{sen} \theta) \operatorname{sen} \theta d \theta=\pi I_{I}(x),
\end{array}\right.
$$

dalle (4) si deduce:

$$
\left\{\begin{array}{l}
\int_{0}^{\pi / 2} I_{0}(x \operatorname{sen} \theta) \operatorname{sen} \theta d \theta=\frac{\operatorname{sen} x}{x} \\
\int_{0}^{\pi / 2} I_{I}(x \operatorname{sen} \theta) d \theta=\frac{1-\cos x}{x} .
\end{array}\right.
$$

(*) Intorno ad un teorema di ABEL $e$ ad alcune sue applicazioni. "Rend. Ist. Lomb.", s. $2^{\text {a }}$, vol. XII (1880); "Opere mat.», t. III, pp. 248-257. [N. d. R.]. 
Come Schlömilch valendosi delle (5) e del teorema d'ABEL ottiene lo sviluppo d'una funzione per funzioni cilindriche d'ordine zero ed uno, cogli argomenti $x, 2 x, 3 x, \cdots$, così il BELtRAMI valendosi delle (6) e del teorema d'ABEL perviene allo sviluppo di una funzione per serie trigonometrica di seni e coseni cogli argomenti $v_{1} x, v_{2} x, v_{3} x, \ldots$, in cui le $v_{i}$ sono le radici positive di una delle equazioni trascendenti $\mathrm{I}_{\mathrm{o}}(x)=0, \mathrm{I}_{\mathrm{o}}^{\prime}(x)=0$.

Infatti, se la funzione $f(x)$ ammette nell'intervallo $(\mathrm{O}, \mathrm{I})$ lo sviluppo:

$$
f(x)=\mathrm{A}_{1} \mathrm{I}_{\mathrm{o}}\left(a_{1} x\right)+\mathrm{A}_{2} \mathrm{I}_{\mathrm{o}}\left(a_{2} x\right)+\cdots,
$$

in cui $a_{1}, a_{2}, \ldots$, sono radici di una o dell'altra equazione $\mathrm{I}_{\mathrm{o}}(x)=0$, $\mathrm{I}_{\circ}^{\prime}(x)=0$, e se al detto sviluppo è applicabile l'integrazione per serie, in virtù di ben note proprietà delle funzioni di BESSEL, si avrà:

$$
\mathrm{A}_{n}=\frac{2}{\mathrm{I}_{0}\left(a_{n}\right)^{2}+\mathrm{I}_{x}\left(a_{n}\right)^{2}} \int_{0}^{\mathrm{I}} x f(x) \mathrm{I}_{0}\left(a_{n} x\right) d x .
$$

Ora sostituiscasi nella (7) $x \operatorname{sen} \theta$ al posto di $x$, si moltiplichi per $\operatorname{sen} \theta d \theta$ e s'integri fra o e $\pi / 2$. A cagione della prima delle (6) si otterrà:

$$
x \int_{0}^{\pi / 2} f(x \operatorname{sen} \theta) \operatorname{sen} \theta d \theta=\Sigma \frac{\mathrm{A}_{n}}{a_{n}} \operatorname{sen} a_{n} x=\mathrm{F}(x) .
$$

Ma il teorema di ABEL ci dà:

$$
f(x)=\frac{1}{\pi} \int_{0}^{\pi} F^{\prime}(x \operatorname{sen} \theta) d \theta
$$

abbiamo dunque il modo di calcolare i coefficienti dello sviluppo (9) per mezzo di $\mathrm{F}(x)$ valendosi della formula precedente insieme alla (8); si trova così:

$$
\mathrm{A}_{n}=\frac{2}{\pi\left[\mathrm{I}_{\circ}\left(a_{n}\right)^{2}+\mathrm{I}_{\mathrm{x}}\left(a_{n}\right)^{2}\right]} \int_{0}^{\mathrm{I}} x \mathrm{I}_{0}\left(a_{n} x\right) d x \int_{0}^{\pi} \mathrm{F}^{\prime}(x \operatorname{sen} \theta) d \theta .
$$

Analogamente se $f_{I}(x)$ ammette lo sviluppo integrabile termine a termine:

$$
f_{x}(x)=\mathrm{B}_{x} \mathrm{I}_{1}\left(b_{1} x\right)+\mathrm{B}_{2} \mathrm{I}_{x}\left(b_{2} x\right)+\mathrm{B}_{3} \mathrm{I}_{x}\left(b_{3} x\right)+\cdots,
$$

essendo le $b_{x}, b_{2}, b_{3}, \ldots$, le radici positive di una delle equazioni $\mathrm{I}_{x}(x)=0$, $\mathrm{I}_{\mathrm{I}}^{\prime}(x)=0$, ossia di una delle due equazioni $\mathrm{I}_{\mathrm{o}}^{\prime}(x)=0, \mathrm{I}_{\mathrm{o}}^{\prime \prime}(x)=0$, operando su questa serie in modo analogo a quello tenuto colla (7) e giovandosi della seconda delle (6) avremo:

$$
x \int_{0}^{\pi / 2} f_{I}(x \operatorname{sen} \theta) d \theta=\Sigma \frac{B_{n}}{b_{n}}\left(\mathrm{I}-\cos b_{n} x\right)=\mathrm{F}_{1}(x),
$$

da cui si ricava pel teorema d'ABEL, mediante un calcolo facile:

$$
f_{I}(x)=\frac{1}{\pi} \int_{0}^{\pi} F_{I}^{\prime}(x \operatorname{sen} \theta) \operatorname{sen} \theta d \theta .
$$


Ma i coefficienti $\mathrm{B}_{n}$ si ottengono da $f_{\mathrm{I}}(x)$ colla formula:

$$
\mathrm{B}_{n}=\frac{2}{\mathrm{I}_{\mathrm{I}}\left(b_{n}\right)^{2}+\mathrm{I}_{2}\left(b_{n}\right)^{2}} \int_{0}^{x} x f_{\mathrm{I}}(x) \mathrm{I}_{\mathrm{I}}\left(b_{n} x\right) d x
$$

quindi essi si ricaveranno, in virtù della (IO) dalla $F_{I}(x)$, e avremo:

$$
\mathrm{B}_{n}=\frac{2}{\pi\left[\mathrm{I}_{\mathrm{I}}\left(b_{n}\right)^{2}+\mathrm{I}_{2}\left(b_{n}\right)^{2}\right]} \int_{0}^{\mathrm{I}} x \mathrm{I}_{\mathrm{I}}\left(b_{n} x\right) d x \int_{0}^{\pi} \mathrm{F}_{\mathrm{I}}^{\prime}(x \operatorname{sen} \theta) \operatorname{sen} \theta d \theta
$$

7. Lo stesso concetto che mosse il BELTRAMI nell'estendere le ricerche dello SchLömILCH, guidò pure il DinI nell'ampliarle a casi più generali.

Nel Cap. VII della sua opera Sulla rappresentazione delle funzioni di una variabile reale, che è stato stampato negli "Annali " delle Università toscane [vol. I 7, parte $2^{\mathrm{a}}$, pp. I-204], il prof. DiNI, dopo avere ottenuto sotto una forma generale lo sviluppo in serie di funzioni reali, passa ad integrare termine a termine le dette serie, dopo averle moltiplicate per una funzione arbitraria. Ottiene in tal modo nuovi sviluppi. Se quello primitivo è

$$
f(x)=\sum_{0}^{\infty} \mathrm{A}_{n} \mathrm{H}_{n}(x)
$$

i cui coefficienti $\mathrm{A}_{n}$ possono determinarsi dipendentemente dalla $f(x)$, e se $\psi(x, \xi)$ è la funzione moltiplicatrice, si ottiene in tal modo lo sviluppo in serie della funzione:

$$
\int_{c}^{\xi} f(x) \psi(x, \xi) d \xi=F(\xi),
$$

il quale sarà della forma:

$$
\sum_{0}^{\infty} \mathrm{A}_{n} \mathrm{~K}_{n}(\xi)
$$

avendo posto:

$$
\mathrm{K}_{n}(\xi)=\int_{c}^{\xi} \mathrm{H}_{n}(x) \psi(x, \xi) d x
$$

Se si potrà ricavare la $f(x)$, quando sia scelta la $\mathrm{F}(\xi)$, avremo il modo di calcolare i coefficienti dello sviluppo in serie (12) mediante la funzione da svilupparsi $\mathrm{F}(\xi)$.

La possibilità dunque della inversione di una equazione funzionale della forma (I I) rende in generale possibile il passaggio da certi sviluppi i cui coefficienti sanno ricavarsi dalla funzione da svolgersi in serie, a nuovi sviluppi i cui coefficienti godono della stessa proprietà. 
Ora ogni qualvolta si sappia trovare, come avviene nel caso di ABEL, una coppia di funzioni $\psi(x, \xi), \theta(y, x)$ tali che

$$
\int_{y}^{\xi} \psi(x, \xi) \theta(y, x) d x=1
$$

sarà possibile di ottenere una delle dette inversioni mediante una espressione della forma:

$$
f(x)=\int_{c}^{x} \mathrm{~F}(\xi) \theta(\xi, x) d \xi .
$$

Il prof. DiNI fa alcune importanti applicazioni, esaminando in particolare la coppia di funzioni:

$$
\begin{gathered}
\psi(x, \xi)=\frac{2}{\pi} \frac{\left(m+n x^{2}\right)^{p}}{\left(m+n x^{2}\right)^{q}\left( \pm \xi^{2} \mp x^{2}\right)^{p}}, \\
\theta(y, x)=\frac{\operatorname{sen} p \pi}{2} x \frac{\left(m+n y^{2}\right)^{1-p}}{\left(m+n x^{2}\right)^{I-q}\left( \pm x^{2} \mp y^{2}\right)^{I-p}}
\end{gathered}
$$

la quale può farsi rientrare nel caso di ABEL, ponendo:

$$
m+n x^{2}=\frac{\mathrm{I}}{u} \quad, \quad m+n y^{2}=\frac{\mathrm{I}}{v} \quad, \quad m+n \xi^{2}=\frac{\mathrm{I}}{w},
$$

in virtù della formula:

$$
\int_{y}^{\xi} \psi(x, \xi) \theta(y, x) d x=\frac{\operatorname{sen} p \pi}{2 \pi} \int_{v}^{w} \frac{d u}{(w-u)^{p}(u-v)^{\mathrm{I}-p}} .
$$

8. Nessuno degli autori fin qui citato aveva mai oltrepassato il limite segna to dalla formula d'ABEL, tantoché essa ha rappresenta to l'unico esempio di inversioni di integrali definiti con limiti variabili fino al 1884 in cui il SoNINE pubblicò negli "Acta Mathematica " [t. 4] la sua Memoria: Sur la généralisation d'une formule d'ABEL. I1 SONINE stesso in un precedente lavoro del 1879: Recherches sur les fonctions cylindriques et le développement des fonctions continues en série ${ }^{(*)}$ si era limitato a ricavare la consueta formula d'ABEL desumendola da alcune interessanti proprietà delle funzioni di BESSEL.

$\mathrm{Ma}$ il teorema che il SonINE ha reso noto nel $\mathrm{I} 884$ e che era stato da lui trovato due anni prima segna un vero e proprio passo nella questione dell'inversione.

Ecco in che cosa consiste il teorema di SonInE. Se $p$ e $q$ sono due numeri positivi la cui somma è eguale all'unità, prendiamo una serie qualunque di potenze:

$$
\mathrm{I}+c_{1} x+c_{2} x^{2}+\cdots=\varphi(x)
$$

$(*)$ "Math. Ann.", Bd. XVI (1880). [N. d. R.]. 
e formiamo lo sviluppo:

$$
\frac{\mathrm{I}}{\varphi(x)}=\mathrm{I}+d_{1} x+d_{2} x^{2}+\cdots
$$

si avrà allora:

$$
\begin{gathered}
\sigma(x)=x^{-p}\left(\frac{\mathrm{I}}{\Gamma(\mathrm{I}-p)}+\frac{c_{1} x}{\Gamma(2-p)}+\frac{c_{2} x^{2}}{\Gamma(3-p)}+\cdots\right), \\
\psi(x)=x^{-q}\left(\frac{\mathrm{I}}{\Gamma(\mathrm{I}-q)}+\frac{d_{\mathrm{I}} x}{\Gamma(2-q)}+\frac{d_{2} x^{2}}{\Gamma(3-q)}+\cdots\right), \\
\int_{a}^{x} f(\xi) d \xi=\int_{a}^{x} \psi(x-\lambda) d \lambda \int_{a}^{\lambda} f(\xi) \sigma(\lambda-\xi) d \xi
\end{gathered}
$$

vale a dire la equazione funzionale:

$$
\mathrm{F}(y)=\int_{a}^{y} \varphi(x) \psi(y-x) d x
$$

si inverte mediante la formula:

$$
\varphi(y)=\int_{a}^{y} F^{\prime}(x) \sigma(y-x) d x .
$$

La formula di SonINE, che diviene quella di ABEL quando la serie $\varphi(x)$ si riduce al suo primo termine, offre quindi infiniti nuovi casi di inversione ottenuti per la prima volta dopo quello di ABEL.

La proprietà caratteristica delle funzioni $\sigma$ e $\psi$ da cui dipende la possibilità della inversione consiste in ciò che:

$$
\mathrm{I}=\int_{x}^{y} \psi(y-\xi) \sigma(\xi-x) d \xi .
$$

Il teorema di SONINE dà quindi infinite soluzioni della equazione funzionale che si era proposta il DINI, ossia dà infinite coppie di funzioni che soddisfano la (13) distinte dalla soluzione di ABEL, e perciò essa offre campo di applicazioni agli sviluppi in serie.

Il SonINE in una Nota del 1889 ne ha fatto anche una applicazione alla riduzione di un integrale multiplo.

Analizzando il processo ideato dal SonINE appare evidente che egli si è proposto di invertire la equazione funzionale (I6) mediante una funzione avente la espressione prestabilita (I7), e ciò per analogia colla formula di ABEL. Anche il DINI era stato condotto a proporsi il problema in maniera analoga. Ma il prestabilire la forma della soluzione ha di necessità ristretto il campo in cui ha potuto applicarsi la inversione stessa, giacché come il SONINE osserva subito nella sua Memoria del I884, egli deve di necessità limitarsi a funzioni $\psi$ e $\sigma$ che divengono infinite allorché il loro argomento si annulla. 
Nella mia Nota II dell'Accademia di Torino risulta il perché in questo caso la forma della soluzione diviene tale, mentre è diversa quando le funzioni sono finite. Nell'Art. III vedremo poi come il teorema di SonINE si deduce dalle formule generali ivi stabilite con principii del tutto differenti. Per la validità del teorema stesso non si richiede poi la convergenza delle serie (14) e (15).

9. I problemi di inversione della equazione funzionale:

$$
f(y)=\int \varphi(x) \mathrm{H}(x, y) d x
$$

con $\varphi(x)$ funzione incognita, allorché i limiti dell'integrale anziché esser variabili sono costanti, hanno dato luogo a studii di una natura totalmente diversa da quelli fino ad ora esaminati, fra cui citeremo quelli del prof. PinCHERLE. Noi non staremo a parlarne giacché le due questioni hanno seguìto cammini differenti tanto che l'una non ha, che io sappia, contribuito fino ad ora all'avanzamento dell'altra.

Di ricerche che legano in certo modo fra loro le due specie di questioni è a mia conoscenza, oltre ad una mia breve Nota del 1884: Sopra un problema di elettrostatica ${ }^{(*)}$, una Nota del prof. Levi-CivitA, dei cui risultati lo stesso autore si è valso in una questione di fisica matematica.

10. Nelle quattro Note che ho presentate all'Accademia di Torino, e nelle due che ho comunicate a quella dei Lincei citate precedentemente, ho cercato una esposizione sistematica della teoria dell'inversione degl'integrali definiti ottenendo le condizioni in cui il problema, allorché i limiti sono $a$ ed $x$, è possibile e determinato e risolvendolo effettivamente in generale senza porre restrizioni alla forma delle funzioni date ed estendendolo poscia al caso di più funzioni incognite e di funzioni di più variabili.

Il metodo che ho seguito è fondato sopra tre principii fondamentali.

PRINCIPIO DI CONVERGENZA. - Se $S_{\circ}(x, y), a<x<y, a<y<b$ è una funzione finita integrabile ${ }^{(4)}$ e a partire da essa si calcolano successivamente le:

$$
\mathrm{S}_{i}(x, y)=\int_{x}^{y} \mathrm{~S}_{i-j}(x, \xi) \mathrm{S}_{j-1}(\xi, y) d \xi,
$$

$S_{i}$ sarà indipendente da $j$ e la serie:

$$
s_{\mathrm{o}}(x, y)=-\sum_{0}^{\infty} \mathrm{S}_{i}(x, y),
$$

sarà uniformemente convergente e rappresenterà una funzione integrabile.

$\left(^{*}\right)$ In queste "Opere »: vol. primo, XI, pp. I88-195.

(4) Nella condizione di integrabilità includiamo quella della possibilità della inversione dell'ordine d'integrazione rispetto alle variabili $x, y$. 
PRINCIPIO DI RECIPROCITÀ. - Se si opera sulla $s_{\mathrm{o}}(x, y)$ come si è operato sulla $\mathrm{S}_{\mathrm{o}}(x, y)$, ciò̀ se si forma:

$$
s_{i}(x, y)=\int_{x}^{y} s_{i-j}(x, \xi) s_{j-\mathrm{I}}(\xi, y) d \xi,
$$

si ritrova la funzione primitiva, vale a dire si ha:

$$
\mathrm{S}_{\mathrm{o}}(x, y)=-\sum_{0}^{\infty} s_{i}(x, y),
$$

e le due funzioni $\mathrm{S}_{\mathrm{o}}(x, y)$ e $s_{\mathrm{o}}(x, y)$ sono legate dalla relazione:

$$
\mathrm{S}_{\mathrm{o}}(x, y)+s_{\mathrm{o}}(x, y)=\int_{x}^{y} \mathrm{~S}_{\mathrm{o}}(x, \xi) s_{\mathrm{o}}(\xi, y) d \xi=\int_{x}^{y} s_{\circ}(x, \xi) \mathrm{S}_{\mathrm{o}}(\xi, y) d \xi .
$$

PRINCIPIO DELLA INVERSIONE. - L'equazione funzionale:

$$
f(y)=\varphi(y)-\int_{a}^{y} \varphi(x) \mathrm{S}_{\mathrm{o}}(x, y) d x
$$

si inverte in una maniera unica mediante la formula:

$$
\varphi(y)=f(y)-\int_{a}^{y} f(x) s_{\mathrm{o}}(x, y) d x .
$$

Questi tre principii sono estensibili al caso di un sistema di funzioni, vale a dire $s e$, invece della sola funzione $\mathrm{S}_{\mathrm{o}}(x, y)$, si hanno le $n^{2}$ funzioni $\mathrm{S}_{r, t}^{(\circ)}(x, y)$, in cui gl'indici $r, t$ prendono $i$ valori $\mathrm{I}, 2, \cdots, n$, e a partire $d a$ esse si calcolano successivamente le:

$$
\mathrm{S}_{r, t}^{(i)}(x, y)=\int_{x}^{y} \sum_{x}^{n} \mathrm{~S}_{r, h}^{(i-j)}(x, \xi) \mathrm{S}_{h, t}^{(j-1)}(\xi, y) d \xi
$$

queste non dipendono dall'indice $j$, e le serie:

$$
s_{r, t}^{(o)}(x, y)=-\sum_{o}^{\infty} S_{r, t}^{(i)}(x, y),
$$

sono uniformemente convergenti. Inoltre ripetendo sulle $s_{r, t}^{(o)}(x, y)$ le operazioni eseguite sulle $\mathrm{S}_{r, t}^{(\circ)}(x, y)$ si ritrovano le funzioni primitive $\mathrm{S}_{r, t}^{(\circ)}(x, y)$, cioè:

$$
\mathrm{S}_{r, t}^{(o)}(x, y)=-\sum_{\mathrm{I}}^{\infty} s_{r, t}^{(i)}(x, y)
$$

essendo:

$$
s_{r, i}^{(i)}(x, y)=\int_{x}^{y} \sum_{x}^{n} s_{r, h}^{(i-j)}(x, \xi) s_{h, t}^{(j-x)}(\xi, y) d \xi
$$


e si ha:

$$
\begin{aligned}
\mathrm{S}_{r, t}^{(o)}(x, y)+s_{r, t}^{(\circ)}(x, y) & =\int_{x}^{y} \sum_{x}^{n} \mathrm{~S}_{r, h}^{(\circ)}(x, \xi) s_{h, t}^{(\circ)}(\xi, y) d \xi \\
& =\int_{x}^{y} \sum_{x}^{n} s_{r, h}^{(\circ)}(x, \xi) \mathrm{S}_{h, t}^{(o)}(\xi, y) d \xi, \quad \text { (principio di reciprocità). }
\end{aligned}
$$

Finalmente vale il principio dell'inversione; ossia: il sistema di equazioni funzionali:

$$
f_{i}(y)=\varphi_{i}(y)-\sum_{I}^{n} \int_{x}^{y} S_{i, h}^{(o)}(x, y) \varphi_{h}(x) d x, \quad(i=\mathrm{I}, 2, \ldots, n)
$$

si inverte in una maniera unica mediante le formule:

$$
\varphi_{i}(y)=f_{i}(y)-\sum_{1}^{n} \int_{a}^{y} s_{i, h}^{(o)}(x, y) f_{h}(x) d x, \quad(i=\mathrm{I}, 2, \cdots, n) .
$$

Una ulteriore estensione può poi ottenersi considerando, invece che una funzione o un sistema di funzioni di una coppia di variabili, una funzione o un sistema di funzioni di $m$ coppie di variabili:

$\mathrm{Se}$

$\left.\mathrm{S}_{\circ}\left(x_{1}, x_{2}, \cdots, x_{n}\right) \mid y_{1}, y_{2}, \cdots, y_{n}\right) \quad, \quad a_{i}<x_{i}<y_{i} \quad, \quad a_{i}<y_{i}<b_{i}$,

è una funzione integrabile, le:

$$
\begin{gathered}
\mathrm{S}_{i}\left(x_{\mathrm{I}}, \cdots, x_{n} \mid y_{1}, \ldots, y_{n}\right) \\
=\int_{x_{\mathrm{I}}}^{y_{1}} d \xi_{\mathrm{I}} \cdots \int_{x_{n}}^{y_{n}} d \xi_{n} \mathrm{~S}_{i-j}\left(x_{\mathrm{I}}, \cdots, x_{n} \mid \xi_{1}, \cdots, \xi_{n}\right) \mathrm{S}_{j-1}\left(\xi_{\mathrm{I}}, \cdots, \xi_{n} \mid y_{1}, \cdots, y_{n}\right)
\end{gathered}
$$

saranno indipendenti dall'indice j; la serie;

$$
s_{\circ}\left(x_{1}, \cdots, x_{n} \mid y_{x}, \cdots, y_{n}\right)=-\sum_{0}^{\infty} \mathrm{S}_{i}\left(x_{1}, \cdots, x_{n} \mid y_{1}, \ldots, y_{n}\right),
$$

sarà uniformemente convergente, e, posto:

$$
\begin{gathered}
s_{i}\left(x_{1}, \cdots, x_{n} \mid y_{1}, \ldots, y_{n}\right) \\
=\int_{x_{x}}^{y_{1}} d \xi_{1} \ldots \int_{x_{n}}^{y_{n}} d \xi_{n} s_{i-j}\left(x_{1}, \cdots, x_{n} \mid \xi_{1}, \ldots, \xi_{n}\right) s_{j-1}\left(\xi_{1}, \ldots, \xi_{n} \mid y_{x}, \cdots, y_{n}\right),
\end{gathered}
$$

risulterà (principio di reciprocità):

$$
\mathrm{S}_{\mathrm{o}}\left(x_{1}, \cdots, x_{n} \mid y_{1}, \cdots, y_{n}\right)=-\sum_{0}^{\infty} s_{i}\left(x_{1}, \cdots, x_{n} \mid y_{1}, \cdots, y_{n}\right)
$$


mentre:

$$
\begin{aligned}
& \mathrm{S}_{\mathrm{O}}\left(x_{\mathrm{I}}, \cdots, x_{n} \mid y_{\mathrm{I}}, \cdots, y_{n}\right)+s_{\mathrm{O}}\left(x_{\mathrm{I}}, \cdots, x_{n} \mid y_{\mathrm{I}}, \cdots, y_{n}\right) \\
= & \int_{x_{\mathrm{I}}}^{y_{\mathrm{I}}} d \xi_{1} \cdots \int_{x_{n}}^{y_{n}} d \xi_{n} \mathrm{~S}_{\mathrm{O}}\left(x_{\mathrm{I}}, \cdots, x_{n} \mid \xi_{\mathrm{I}}, \cdots, \xi_{n}\right) s_{\mathrm{O}}\left(\xi_{\mathrm{I}}, \cdots, \xi_{n} \mid y_{\mathrm{I}}, \cdots, y_{n}\right) \\
= & \int_{x_{\mathrm{I}}}^{y_{\mathrm{I}}} d \xi_{\mathrm{I}} \cdots \int_{x_{n}}^{y_{n}} d \xi_{n} s_{\mathrm{O}}\left(x_{\mathrm{I}}, \cdots, x_{n} \mid \xi_{\mathrm{I}}, \cdots, \xi_{n}\right) \mathrm{S}_{\mathrm{O}}\left(\xi_{\mathrm{I}}, \cdots, \xi_{n} \mid y_{1}, \cdots, y_{n}\right) ;
\end{aligned}
$$

e. l'equazione (principio della inversione):

$$
\begin{gathered}
f\left(y_{1}, \cdots, y_{n}\right)=\varphi\left(y_{1}, \cdots, y_{n}\right) \\
-\int_{a_{1}}^{y_{1}} d x_{1} \cdots \int_{a_{n}}^{y_{n}} d x_{n} \varphi\left(x_{1}, \cdots, x_{n}\right) \mathrm{S}_{\mathrm{\circ}}\left(x_{\mathrm{I}}, \cdots, x_{n} \mid y_{\mathrm{I}}, \cdots, y_{n}\right),
\end{gathered}
$$

si invertirà in maniera unica mediante l'altra:

$$
\begin{gathered}
\varphi\left(y_{\mathrm{I}}, \cdots, y_{n}\right)^{\circ}=f\left(y_{\mathrm{I}}, \cdots, y_{n}\right) \\
-\int_{a_{\mathrm{I}}}^{y_{1}} d x_{\mathrm{I}} \cdots \int_{a_{n}}^{y_{n}} d x_{n} f\left(x_{\mathrm{I}}, \cdots, x_{n}\right) s_{\mathrm{O}}\left(x_{\mathrm{I}}, \cdots, x_{n} \mid y_{\mathrm{I}}, \cdots, y_{n}\right) .
\end{gathered}
$$

I I. Con questi principii si riconduce la risoluzione dei vari problemi di inversione, qualunque sia il numero delle funzioni incognite e qualunque sia il numero delle variabili da cui esse dipendono, ad operazioni successive di quadratura.

Nel caso più semplice che possa presentarsi si ottiene il teorema:

Se si ha la equazione funzionale:

$$
f(y)-f(\alpha)=\int_{\alpha}^{y} \varphi(x) \mathrm{H}(x, y) d x,
$$

in cuif $f(y)$ e $f^{\prime}(y)$ si mantengono finite e continue per y compreso fra $\alpha e \alpha+\mathrm{A}$ $e \mathrm{H}(x, y)$ e $\mathrm{\partial H} / \partial y=\mathrm{H}_{2}(x, y)$ sono pure finite per $y>x>\alpha, \alpha+\mathrm{A}>y>\alpha$, e quest'ultima è integrabile, mentre è maggiore di zero il limite inferiore dei valori assoluti di $h(y)=\mathrm{H}(y, y)$, esisterà una ed una sola funzione finita $e$ continua $\varphi$ che soddisfa l'equazione funzionale per y compreso fra $\alpha e \alpha+\mathrm{A}$, la quale sarà data da:

$$
\varphi(y)=\frac{f^{\prime}(y)}{h(y)}-\frac{1}{h(y)} \int_{\alpha}^{y} f^{\prime}(x) \sum_{0}^{\infty} \mathrm{S}_{i}(x, y) d x,
$$

in cui:

$$
\begin{aligned}
& \mathrm{S}_{i}(x, y)=\int_{y}^{x} \mathrm{~S}_{i-j}(x, \xi) \mathrm{S}_{j-1}(\xi, y) d \xi \\
& \mathrm{S}_{\mathrm{o}}(x, y)=\frac{\mathrm{H}_{2}(x, y)}{h(x)} .
\end{aligned}
$$


Nel caso particolare in cui $\mathrm{H}(x, y)$ assume la forma $\mathrm{F}[\lambda(x)-\lambda(y)]$ questo teorema si specializza e diviene:

La formula di inversione della

$$
f(y)-f(\alpha)=\int_{\alpha}^{y} \varphi(x) \mathrm{F}[\lambda(x)-\lambda(y)] d x \quad, \quad[\mathrm{~F}(0)=\mathrm{I}],
$$

$\grave{e}$

$$
\varphi(y)=f^{\prime}(y)+\lambda^{\prime}(y) \int_{\alpha}^{y} f^{\prime}(x) \Theta[\lambda(x)-\lambda(y)] d x,
$$

in cui:

$$
\left\{\begin{array}{l}
\Theta(z)=\sum_{0}^{\infty}(-\mathrm{I})^{i} s_{i}(z) \\
s_{0}(z)=\mathrm{F}(z) \\
s_{i}(z)=\int_{0}^{z} s_{i-j}(z-u) s_{j-I}(u) d u .
\end{array}\right.
$$

Il caso in cui $\mathrm{H}(x, y)$ diviene infinito per $x=y$, in modo che si possa porre $\mathrm{H}(x, y)=\mathrm{G}(x, y) /(x-y)^{\lambda}$ con $\mathrm{G}(x, y)$ finita e $\lambda<\mathrm{I}$, e che comprende in sè evidentemente il caso di SONINE e quindi quello d'ABEL, si può ricondurre, con uno speciale artificio all'analisi precedente, ed in tal modo si ottiene il teorema:

Se si ha l'equazione funzionale:

$$
f(y)-f(\alpha)=\int_{\alpha}^{y} \varphi(x) \frac{\mathrm{G}(x, y)}{(y-x)^{\lambda}} d x \quad, \quad(\lambda<\mathrm{I}),
$$

in cui $f(y)$ e $f^{\prime}(y)$ si mantengono finite e continue per $y$ compreso fra $\alpha e$ $\alpha+\mathrm{A}(\mathrm{A}>0), e \mathrm{G}(x, y)$ e $\mathrm{aG} / \mathrm{\partial} y=\mathrm{G}_{2}(x, y)$ sono pure finite e continue per tutti $i$ valori di $x, y$, compresi entro $i$ limiti $\alpha e \alpha+\mathrm{A}$, mentre è maggiore di zero il limite inferiore dei valori assoluti di $g(y)=\mathrm{G}(y, y)$ per y compreso nello stesso intervallo, esisterà una ed una sola funzione finita e continua $\varphi$ che soddisfa l'equazione funzionale per y compreso fra $\alpha e \alpha+\mathrm{A}$, la quale sarà data da:

$$
\varphi(z)=\frac{\operatorname{sen} \lambda \pi}{\pi} \frac{1}{g(z)} \int_{\alpha}^{z} f^{\prime}(x) \sum_{0}^{\infty} T_{i}(x, y) d x
$$

in cui:

$$
\begin{aligned}
& \mathrm{S}_{\mathrm{o}}(y, z)=\frac{\operatorname{sen} \lambda \pi}{\pi} \frac{\mathrm{I}}{g(z)} \int_{y}^{z} \mathrm{G}_{2}(y, \xi)\left(\frac{\xi-y}{z-\xi}\right)^{\mathrm{x}-\lambda} \frac{d \xi}{z-y}, \\
& \mathrm{~T}_{\mathrm{o}}(x, z)=\frac{1}{(z-x)^{\mathrm{x}-\lambda}}, \\
& \mathrm{T}_{i}(x, z)=\int_{z}^{x} \mathrm{~S}_{\mathrm{o}}(\xi, z) \mathrm{T}_{i-1}(x, \xi) d \xi .
\end{aligned}
$$


Questa proposizione, allorché $\mathrm{G}(x, y)$ ha la forma $\mathrm{F}(y-x)$, si specializza e si riduce all'altra:

La formula di inversione della relazione funzionale:

$$
f(y)-f(\alpha)=\int_{\alpha}^{y} \varphi(x) \frac{\mathrm{F}(y-x)}{(y-x)^{\lambda}} d x \quad, \quad[0<\lambda<\mathrm{I}, \mathrm{F}(\mathrm{o})=\mathrm{I}],
$$

$\grave{e}$

$$
\varphi(z)=\frac{\operatorname{sen} \lambda \pi}{\pi} \int_{\alpha}^{z} f^{\prime}(x) \Omega(z-x) d x
$$

in cui:

$$
\left\{\begin{array}{l}
s_{\mathrm{o}}(v)=\frac{\operatorname{sen} \lambda \pi}{\pi v} \int_{0}^{v} \mathrm{~F}^{\prime}(u)\left(\frac{u}{v-u}\right)^{\mathrm{I}-\lambda} d u \\
t_{\mathrm{o}}(v)=\frac{\mathrm{I}}{v^{\mathrm{I}-\lambda}} \\
t_{i}(v)=\int_{0}^{v} s_{\mathrm{o}}(v-u) t_{i-\mathrm{I}}(u) d u \\
\Omega(v)=\sum_{0}^{\infty}(-\mathrm{I})^{i} t_{i}(v) .
\end{array}\right.
$$

In questo teorema non è necessario, come in quello di SonIne, partire dallo sviluppo in serie di potenze della funzione $\mathrm{F}$.

Finalmente se $\mathrm{H}(x, y)$ si annulla per $x=y$, il problema dell'inversione può in taluni casi riescire determinato, in altri no, e la discriminazione di essi può ricondursi ad operazioni algebriche. Il teorema fondamentale che si ha a questo proposito è il seguente.

Abbiasi la equazione funzionale:

$$
f(y)=\int_{0}^{y} \varphi(x) \mathrm{H}(x, y) d x \quad, \quad a>y>0,
$$

in cui:

$$
\begin{gathered}
f(y)=y^{n+1} f_{1}(y) \\
H(x, y)=\sum_{0}^{n} a_{i} x^{i} y^{n-i}+\sum_{0}^{n+1} x^{i} y^{n+1-i} \mathrm{~L}_{i}(x, y),
\end{gathered}
$$

essendo le $a_{i}$ quantità costanti.

Se $f_{\mathrm{I}}(y)$ e $\mathrm{L}_{i}(x, y)$ e le loro derivate rapporto ad $y$ sono finite e continue per $x$ compreso fra o e y e y compreso fra o ed a, mentre in questo intervallo $h(y)=\mathrm{H}(y, y)$ non si annulla che per $y=0$, esisterà una ed una sola funzione finita e continua che soddisfa la (25) quando tutte le radici dell' equazione algebrica di grado $n$ :

$$
\frac{a_{\circ}}{\lambda-1}+\frac{a_{1}}{\lambda-2}+\cdots+\frac{a_{n}}{\lambda-n-1}=0
$$


essendo finite e differenti fra loro, avranno le parti reali positive. Invece, se le radici della equazione precedente saranno finite e diverse fra loro, ma una o più di esse avranno la parte reale negativa, allora il problema di dedurre $\varphi(x)$ dalla (25) sarà indeterminato.

L'effettiva risoluzione della equazione funzionale (25), quando le condizioni stabilite dal precedente teorema, affinché il problema sia determinato, sono soddisfatte può eseguirsi riconducendo la questione ad un'altra analoga per la quale sia verificata la condizione $\mathrm{H}(y, y) \gtrless 0$. [Vedi perciò la $3^{\mathrm{a}} \mathrm{e}$ la $4^{\text {a }}$ delle mie Note dell'Accademia di Torino].

ART. II.

I. Il metodo precedentemente esposto può estendersi in modo da renderlo applicabile al caso della inversione, quando ambedue i limiti sono variabili. È ciò che mostreremo in questo articolo.

Cominciamo dal ricordare alcune formule ben semplici del calcolo delle differenze finite ${ }^{(5)}$.

Se $\lambda(x)$ e $\varphi(x)$ sono funzioni finite e continue nell'intervallo $(0, a)$ $e|\lambda(\mathrm{O})| \leqq \mathrm{I} e \mathrm{o}<\alpha<\mathrm{I}$, la serie:

$$
\begin{aligned}
\theta(x)= & \varphi(x)+\alpha \lambda(x) \varphi(\alpha x)+\alpha^{2} \lambda(\alpha x) \lambda(x) \varphi\left(\alpha^{2} x\right) \\
& +\alpha^{3} \lambda\left(\alpha^{2} x\right) \lambda(\alpha x) \lambda(x) \varphi\left(\alpha^{3} x\right)+\cdots
\end{aligned}
$$

sarà convergente uniformemente nell'intervallo $(\mathrm{o}, a)$ e la somma $\theta(x)$ sarà una funzione finita e continua.

Infatti sia $M_{n}$ il limite superiore dei valori assoluti di $\lambda(x)$ nell'intervallo $\left(\mathrm{o}, a \alpha^{n}\right)$, ed $\mathrm{M}$ il limite superiore dei valori assoluti di $\varphi(x)$; il termine

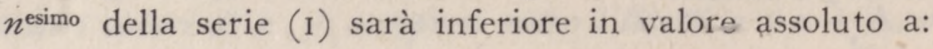

$$
\alpha^{n} M_{o} M_{1} \cdots M_{n-1} M
$$

Ora la serie:

$$
M+\alpha M_{0} M+\alpha^{2} M_{o} M_{1} M+\alpha^{3} M_{0} M_{1} M_{2} M+\cdots,
$$

è convergente, giacché il rapporto fra l' $(n+\mathrm{I})^{\text {esimo }}$ termine e il termine $n^{\text {esimo }}$ è:

$$
\alpha \mathrm{M}_{n}
$$

il quale per $n=\infty$ avrà un limite inferiore all'unità.

La serie (I) converge dunque uniformemente, e siccome ogni termine ̀̀ una funzione finita e continua di $x, \operatorname{così} \theta(x)$ sarà una funzione pure finita e continua. seguenti.

(5) Cfr. Boole, A Treatise on the calculus of finite differences. Ch. IX, Art. 6 e 
Questo teorema può estendersi al caso in cui $\alpha$ sia negativo ma sempre tale che $|\alpha|<\mathrm{I}$, in modo che si ha il teorema:

Se $\lambda(x)$ e $\varphi(x)$ sono funzioni finite e continue nell'intervallo $(\alpha a, a) e$ $|\lambda(\mathrm{O})| \leqq \mathrm{I}, \mathrm{O}>\alpha>-\mathrm{I}$, la serie (I) sarà convergente uniformemente nell'intervallo $(\alpha a, a)$ e rappresenterà una funzione finita e continua.

$\mathrm{Ci}$ si può ora proporre il problema di trovare la funzione $\varphi(x)$ quando sia data la funzione $\theta(x)$.

Abbiamo:

$$
\theta(\alpha x)=\varphi(\alpha x)+\alpha \lambda(\alpha x) \varphi\left(\alpha^{2} x\right)+\alpha^{2} \lambda\left(\alpha^{2} x\right) \lambda(\alpha x) \varphi\left(\alpha^{3} x\right)+\cdots,
$$

quindi:

$$
\theta(x)-\alpha \lambda(x) \theta(\alpha x)=\varphi(x),
$$

e questa formula risolve la questione proposta.

Reciprocamente la (I) risolve il problema di determinare $\theta(x)$ quando si conosce $\varphi(x)$, onde possiamo considerare le due formole (I) e (2) come inverse o reciproche l'una dell'altra, per modo che se $\lambda(x)$ è finita e continua $e|\lambda(\mathrm{O})| \leq \mathrm{I},|\alpha|<\mathrm{I}$, scelta arbitrariamente la funzione finita e continua $\theta(x)$ la (2) ci darà $\varphi(x)$, mentre scelta aróitrariamente la funzione finita e continua $\varphi(x)$, la $(\mathrm{I})$ ci darà $\theta(x)$. Se $\alpha>0$ basìerà considerare $\lambda(x), \theta(x)$ e $\varphi(x)$ in un intervallo $(0, a)$, mentre se $\alpha<0$ dovremo considerarle in un intervallo $(\alpha a, a)$.

2. Ciò premesso, abbiasi la equazione funzionale:

$$
f(y)-f(0)=\int_{\alpha y}^{y} \theta(x) \mathrm{H}(x, y) d x \quad, \quad(a>y>0),
$$

in cui $\mathrm{I}>\alpha>0$ e $f(y), f^{\prime}(y), \mathrm{H}(x, y), \mathrm{H}_{2}(x, y)=\partial \mathrm{H} / \mathrm{c} y$ sono funzioni finite e continue per $x, y$ variabili rispettivamente negl'intervalli $(\alpha y, y)(0, a)$ e $\theta(x)$ è una funzione incognita nell'intervallo $(0, a)$.

Derivando la precedente equazione, avremo:

$$
f^{\prime}(y)=\theta(y) \mathrm{H}(y, y)-\alpha \theta(\alpha y) \mathrm{H}(\alpha y, y)+\int_{\alpha y}^{y} \theta(x) \mathrm{H}_{2}(x, y) d x,
$$

e se il limite inferiore dei valori assoluti di $\mathrm{H}(y, y)$ è diverso da zero:

Posto:

$$
\frac{f^{\prime}(y)}{\mathrm{H}(y, y)}=\theta(y)-\alpha \theta(\alpha y) \frac{\mathrm{H}(\alpha y, y)}{\mathrm{H}(y, y)}+\int_{\alpha y}^{y} \theta(x) \frac{\mathrm{H}_{2}(x, y)}{\mathrm{H}(y, y)} d x .
$$

$$
\frac{f^{\prime}(y)}{\mathrm{H}(y, y)}=\psi(y) \quad, \quad \frac{\mathrm{H}(\alpha y, y)}{\mathrm{H}(y, y)}=\lambda(y) \quad, \quad \frac{\mathrm{H}_{2}(x, y)}{\mathrm{H}(y, y)}=\mathrm{L}(x, y),
$$

avremo:

$$
\psi(y)=\theta(y)-\alpha \lambda(y) \theta(\alpha y)+\int_{\alpha y}^{y} \theta(x) \mathrm{L}(x, y) d x
$$


onde, ponendo $\varphi(y)=\theta(y)-\alpha \lambda(y) \theta(\alpha y)$, e quindi, ricorrendo alla (I), si otterrà:

$\psi(y)=\varphi(y)+\int_{\alpha y}^{y}\left\{\varphi(x)+\alpha \lambda(x) \varphi(\alpha x)+\alpha^{2} \lambda(x) \lambda(\alpha x) \varphi\left(\alpha^{2} x\right)+\cdots\right\} \mathrm{L}(x, y) d x$, ove, a cagione della convergenza in egual grado della serie contenuta sotto il segno d'integrazione:

$$
\begin{aligned}
\psi(y)=\varphi & (y)+\int_{\alpha y}^{y} \varphi(x) \mathrm{L}(x, y) d x+\int_{\alpha y}^{y} \varphi(\alpha x) \alpha \lambda(x) \mathrm{L}(x, y) d x \\
& +\int_{\alpha y}^{y} \varphi\left(\alpha^{2} x\right) \alpha^{2} \lambda(x) \lambda(\alpha x) \mathrm{L}(x, y) d x+\cdots
\end{aligned}
$$

Se ora nel secondo integrale del secondo membro sostituiamo $x$ ad $\alpha x$, nel terzo sostituiamo $x$ ad $\alpha^{2} x$ e cosi di seguito, la precedente equazione si scriverà:

$$
\begin{aligned}
\psi(y)=\varphi(y) & +\int_{\alpha y}^{y} \varphi(x) \mathrm{L}(x, y) d x+\int_{\alpha^{2} y}^{\alpha y} \varphi(x) \lambda\left(\frac{x}{\alpha}\right) \mathrm{L}\left(\frac{x}{\alpha}, y\right) d x \\
& +\int_{\alpha^{3} y}^{\alpha y} \varphi(x) \lambda\left(\frac{x}{\alpha^{2}}\right) \lambda\left(\frac{x}{\alpha}\right) \mathrm{L}\left(\frac{x}{\alpha^{2}}, y\right) d x+\cdots
\end{aligned}
$$

3. Si immagini il triangolo OAB compreso fra l'asse $y$, la bisettrice dell'angolo degli assi, $x, y$ e la retta $y=a$, quindi si tirino le rette

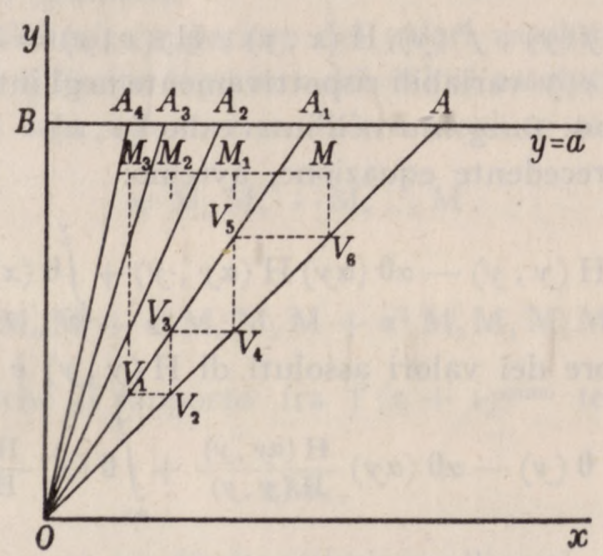

(Nella figura si è supposto $n=3$ ).

$\mathrm{OA}_{1}, \mathrm{OA}_{2}, \mathrm{OA}_{3}, \cdots$, tali che formino con $y$ angoli le cui tangenti trigonometriche siano successivamente $\alpha, \alpha^{2}, \alpha^{3}, \ldots$ Verrà così diviso il triangolo $\mathrm{OAB}$ in un numero infinito di triangoli $\mathrm{OAA}_{1}, \mathrm{OA}_{1} \mathrm{~A}_{2}, \mathrm{OA}_{2} \mathrm{~A}_{3}, \cdots$ Ammetteremo che $\mathrm{i}$ punti di ciascun lato $\mathrm{OA}_{n}$ appartengano al triangolo $\mathrm{OA}_{n-1} \mathrm{~A}_{n}$ 
e non al triangolo successivo $\mathrm{OA}_{n} \mathrm{~A}_{n+\mathrm{r}}$; soltanto il lato $\mathrm{OA}$ lo supporremo appartenere al triangolo $\mathrm{OAA}_{\mathrm{x}}$. In tal modo scelto un punto qualsiasi del triangolo $\mathrm{OAB}$ (escluso il lato $\mathrm{OB}$ ) esso apparterrà ad uno e ad uno solo dei triangoli precedentemente costrutti. Appartenga il punto scelto al triangolo $\mathrm{OA}_{n} \mathrm{~A}_{n+1}$, denotiamolo con $\mathrm{M}_{n}$ e chiamiamo $x, y$ le sue coordinate. I punti $\mathrm{M}_{n-1}, \mathrm{M}_{n-2}, \cdots, \mathrm{M}_{2}, \mathrm{M}_{1}, \mathrm{M}$ aventi rispettivamente le coordinate:

$$
\left(\frac{x}{\alpha}, y\right),\left(\frac{x}{\alpha^{2}}, y\right), \cdots\left(\frac{x}{\alpha^{n-2}}, y\right),\left(\frac{x}{\alpha^{n-1}}, y\right),\left(\frac{x}{\alpha^{n}}, y\right),
$$

apparterranno ordinatamente ai triangoli:

$$
\mathrm{OA}_{n-1} \mathrm{~A}_{n}, \mathrm{OA}_{n-2} \mathrm{~A}_{n-1}, \cdots, \mathrm{OA}_{2} \mathrm{~A}_{3}, \mathrm{OA}_{\mathrm{I}} \mathrm{A}_{2}, \mathrm{OAA}_{1},
$$

e si potranno chiamare corrispondenti di $\mathrm{M}_{n}$.

Scelto $M_{n}$ sarà facile ottenere $M$ mediante una costruzione geometrica. Basterà tirare da $\mathrm{M}_{n}$ la parallela all'asse $y$ finché non si incontra il lato $\mathrm{OA}_{x}$ e a partire da questo punto d'intersezione $V_{I}$ costruire una spezzata:

$$
\mathrm{V}_{1} \mathrm{~V}_{2} \mathrm{~V}_{3} \ldots \mathrm{V}_{2 n-1} \mathrm{~V}_{2 n} \mathrm{M}
$$

internamente al triangolo $\mathrm{OAA}_{\mathrm{I}}$, avente $\mathrm{i}$ vertici $\mathrm{V}_{\mathrm{I}}, \mathrm{V}_{3}, \ldots, \mathrm{V}_{2 n-\mathrm{I}}$ sul lato $\mathrm{OA}_{1}$ e $\mathrm{i}$ vertici $\mathrm{V}_{2}, \mathrm{~V}_{4}, \ldots, \mathrm{V}_{2 n}$ sul lato $\mathrm{OA}$ ed $\mathrm{i}$ lati successivamente paralleli agli assi $x, y$ finché non si incontra in M la parallela ad $x$ condotta dal punto $\mathrm{M}_{n}$.

4. Per ciò che è stato detto nel $\S 2, \mathrm{H}(x, y)$ e quindi $\mathrm{L}(x, y)$ sono funzioni definite nei punti del triangolo $\mathrm{OAA}_{x}$, mentre $\lambda(y)$ è definita nell'intervallo $(\mathrm{o}, a)$. Si prolunghi ora la funzione $\mathrm{L}(x, y)$ in tutto lo spazio $\mathrm{OAB}$ (escluso il lato $\mathrm{OB}$ ), prendendone il valore in un punto qualsiasi $\mathrm{M}_{n}$ dato da:

$$
\lambda\left(\frac{x}{\alpha}\right) \lambda\left(\frac{x}{\alpha^{2}}\right) \cdots \lambda\left(\frac{x}{\alpha^{n}}\right) \mathrm{L}\left(\frac{x}{\alpha^{n}}, y\right),
$$

e denotiamo con $\mathrm{K}(x, y)$ la funzione così ottenuta. Si riconosce subito che essa sarà in generale discontinua lungo le linee $\mathrm{OA}_{1}, \mathrm{OA}_{2}, \ldots, \mathrm{OA}_{n}, \ldots$

Il suo valore potrà essere scritto, in virtù delle (4), sotto la forma:

$$
\mathrm{K}(x, y)=\frac{\mathrm{H}\left(x, \frac{x}{\alpha}\right)}{\mathrm{H}\left(\frac{x}{\alpha}, \frac{x}{\alpha}\right)} \cdot \frac{\mathrm{H}\left(\frac{x}{\alpha}, \frac{x}{\alpha^{2}}\right)}{\mathrm{H}\left(\frac{x}{\alpha^{2}}, \frac{x}{\alpha^{2}}\right)} \cdots \frac{\mathrm{H}\left(\frac{x}{\alpha^{n-x}}, \frac{x}{\alpha^{n}}\right)}{\mathrm{H}\left(\frac{x}{\alpha^{n}}, \frac{x}{\alpha^{n}}\right)} \mathrm{L}\left(\frac{x}{\alpha^{n}}, y\right),
$$

ossia:

$$
\mathrm{K}\left(\mathrm{M}_{n}\right)=\frac{\mathrm{H}\left(\mathrm{V}_{\mathrm{x}}\right) \mathrm{H}\left(\mathrm{V}_{3}\right) \cdots \mathrm{H}\left(\mathrm{V}_{2} n-1\right)}{\mathrm{H}\left(\mathrm{V}_{2}\right) \mathrm{H}\left(\mathrm{V}_{4}\right) \cdots \mathrm{H}\left(\mathrm{V}_{2 n}\right)} \mathrm{L}(\mathrm{M}),
$$

denotando con $\mathrm{F}(\mathrm{V})$ il valore di una funzione di $x, y$ nel punto $\mathrm{V}$.

Riferendoci quindi alla costruzione geometrica precedentemente indicata, potremo dire che il valore della funzione $\mathrm{K}$ in un punto qualunque del triangolo $\mathrm{OAB}$ (escluso $\mathrm{OB})$ è eguale al valore della funzione $\mathrm{L}$ nel punto corrispondente del triangolo $\mathrm{OAA}_{x}$ moltiplicato per il prodotto dei valori di $\mathrm{H}$ nei 
vertici d'ordine dispari della poligonale costruita e diviso per il prodotto dei valori di $\mathrm{H}$ nei vertici d'ordine pari. È facile dimostrare che $|\mathrm{K}(x, y)|$ sarà sempre inferiore ad un numero finito.

Infatti la (7) potrà scriversi:

$$
\begin{gathered}
\mathrm{K}(x, y)=\frac{\mathrm{H}\left(x, \frac{x}{\alpha}\right)}{\mathrm{H}\left(\frac{x}{\alpha^{n}}, \frac{x}{\alpha^{n}}\right)}\left[\mathrm{I}+\frac{\mathrm{H}_{2}\left(\frac{x}{\alpha}, \xi_{1}\right)}{\mathrm{H}\left(\frac{x}{\alpha}, \frac{x}{\alpha}\right)}\left(\frac{x}{\alpha^{2}}-\frac{x}{\alpha}\right)\right]\left[\mathrm{I}+\frac{\mathrm{H}_{2}\left(\frac{x}{\alpha^{2}}, \xi_{2}\right)}{\mathrm{H}\left(\frac{x}{\alpha^{2}}, \frac{x}{\alpha^{2}}\right)}\left(\frac{x}{\alpha^{3}}-\frac{x}{\alpha^{2}}\right)\right] \cdots \\
\cdots\left[\mathrm{I}+\frac{\mathrm{H}_{2}\left(\frac{x}{\alpha^{n-1}}, \xi_{n-1}\right)}{\mathrm{H}\left(\frac{x}{\alpha^{n-1}}, \frac{x}{\alpha^{n-1}}\right)}\left(\frac{x}{\alpha^{n}}-\frac{x}{\alpha^{n-1}}\right)\right] \mathrm{L}\left(\frac{x}{\alpha^{n}}, y^{\prime}\right),
\end{gathered}
$$

in cui le $\xi_{i}$ sono numeri compresi fra $x / \alpha^{i}$ e $x / \alpha^{i+1}$. Sia ora $m$ il limite superiore dei valori assoluti di $\mathrm{H}(x, y), m^{\prime}$ il limite inferiore dei valori assoluti di $\mathrm{H}(x, x)$ e $m^{\prime \prime}$ il limite superiore dei valori assoluti di $\mathrm{H}_{2}(x, y)$, riferendosi alle (4) avremo:

$$
\begin{gathered}
|\mathrm{K}(x, y)| \leq \frac{m m^{\prime \prime}}{m^{\prime 2}}\left[\mathrm{I}+\frac{m^{\prime \prime}}{m^{\prime}}\left(\frac{x}{\alpha^{2}}-\frac{x}{\alpha}\right)\right]\left[\mathrm{I}+\frac{m^{\prime \prime}}{m^{\prime}}\left(\frac{x}{\alpha^{3}}-\frac{x}{\alpha^{2}}\right)\right] \cdots \\
\cdots\left[\mathrm{I}+\frac{m^{\prime \prime}}{m^{\prime}}\left(\frac{x}{\alpha^{n}}-\frac{x}{\alpha^{n-x}}\right)\right]
\end{gathered}
$$

e siccome $x / \alpha^{n}<a$ :

$$
\begin{aligned}
|\mathrm{K}(x, y)|<\frac{m m^{\prime \prime}}{m^{\prime 2}}\left[\mathrm{I}+\frac{m^{\prime \prime}}{m^{\prime}} a\right]\left[\mathrm{I}+\frac{m^{\prime \prime}}{m^{\prime}} a \alpha\right] \cdots\left[\mathrm{I}+\frac{m^{\prime \prime}}{m^{\prime}} a \alpha^{n-2}\right] \\
<\frac{m m^{\prime \prime}}{m^{\prime 2}} \prod_{0}^{\infty}\left[\mathrm{I}+\rho \alpha^{i}\right]
\end{aligned}
$$

in cui $p=\frac{m^{\prime \prime}}{m^{\prime}} a$. Il che dimostra la proposizione.

5. Riprendiamo ora la formula (6) del $\S 2$, ed osserviamo che:

$$
\begin{array}{ll}
\text { per } \quad y \geqq x \geq \alpha y \text { si ha } \mathrm{L}(x, y)=\mathrm{K}(x, y), \\
\text { " } \alpha y>x \geq \alpha^{2} y \quad \text { " } \quad \lambda\left(\frac{x}{\alpha}\right) \mathrm{L}\left(\frac{x}{\alpha}, y\right)=\mathrm{K}(x, y) ; \\
\text { " } \alpha^{2} y>x \geq \alpha^{3} y \quad \text { " } \quad \lambda\left(\frac{x}{\alpha^{2}}\right) \lambda\left(\frac{x}{\alpha}\right) \mathrm{L}\left(\frac{x}{\alpha^{2}}, y\right)=\mathrm{K}(x, y),
\end{array}
$$

e così di seguito indefinitamente; perciò la formula (6) potrà scriversi:

$$
\psi(y) \varphi(y)+\int_{0}^{y} \varphi(x) \mathrm{K}(x, y) d x,
$$

ovvero, ponendo $\mathrm{K}(x, y)=-\mathrm{S}_{\mathrm{o}}(x, y)$,

$$
\psi(y)=\varphi(y)-\int_{0}^{y} \varphi(x) \mathrm{S}_{0}(x, y) d x,
$$


dalla quale si ricaverà, col processo di inversione esposto nell'Art. I, § IO,

$$
\varphi(y)=\psi(y)-\int_{0}^{y} \psi(x) s_{0}(x, y) d x,
$$

in cui $s_{\mathrm{o}}(x, y)$ potrà calcolarsi con quadrature successive mediante $\mathrm{K}(x, y)$.

Ottenuta in tal maniera $\varphi(x)$, la $(I)$ ci darà la funzione incognita $\theta(x)$.

6. La soluzione del problema propostoci può ottenersi anche in un altro modo come accenneremo ora.

Dalla (5) segue:

$$
\theta(y)-\alpha \lambda(y) \theta(\alpha y)=\psi(y)-\int_{\alpha y}^{y} \theta(x) \mathrm{L}(x, y) d x
$$

onde applicando la (I) avremo:

$$
\begin{gathered}
\theta(y)=\psi(y)+\alpha \lambda(y) \psi(\alpha y)+\alpha^{2} \lambda(\alpha y) \lambda(y) \psi\left(\alpha^{2} y\right)+\cdots-\int_{\alpha y}^{y} \theta(x) \mathrm{L}(x, y) d x \\
-\int_{\alpha^{2} y}^{\alpha \cdot y} \theta(x) \alpha \lambda(y) \mathrm{L}(x, \alpha y) d x-\int_{\alpha 3 y}^{\alpha^{2} y} \theta(x) \alpha^{2} \lambda(\alpha y) \lambda(y) \mathrm{L}\left(x, \alpha^{2} y\right) d x+\cdots
\end{gathered}
$$

e ponendo:

$$
\psi(y)+\alpha \lambda(y) \psi(\alpha y)+\alpha^{2} \lambda(\alpha y) \lambda(y) \psi\left(\alpha^{2} y\right)+\cdots=\chi(y),
$$

dalla equazione precedente risulterà:

$$
\begin{aligned}
\chi(y)= & \theta(y)+\int_{\alpha y}^{y} \theta(x) \mathrm{L}(x, y) d x+\int_{\alpha^{2} y}^{\alpha y} \theta(x) \alpha \lambda(y) \mathrm{L}(x, \alpha y) d x \\
& +\int_{\alpha^{3} y}^{\alpha^{2} y} \theta(x) \alpha^{2} \lambda(\alpha y) \lambda(y) \mathrm{L}\left(x, \alpha^{2} y\right) d x+\cdots
\end{aligned}
$$

Questa formula è analoga alla (6) e da essa potrebbe ricavarsi $\theta(y)$ con un procedimento analogo a quello tenuto nei due paragrafi precedenti per ottenere $\varphi(y)$ dalla (6). Noi non staremo però a svilupparlo, ci varremo piuttosto della formula ora trovata per risolvere la equazione funzionale (3) nel caso di $\alpha$ negativo e minore dell'unità.

7. Prima di procedere alla trattazione di questo caso premettiamo le considerazioni seguenti.

Abbiasi la equazione funzionale:

$$
f(y)=\varphi(y)+\int_{-y}^{y} \varphi(x) \mathrm{K}(x, y) d x \quad, \quad(a>y>-a)
$$


in cui $f(y)$ sia definita nell'intervallo $(-a, a)$ e la funzione finita ed integrabile $\mathrm{K}(x, y)$ per $y>x>-y$ e $a>y>-a$. L'equazione precedente potrà scriversi:

$$
f(y)=\varphi(y)+\int_{0}^{y} \varphi(x) \mathrm{K}(x, y) d x+\int_{0}^{y} \varphi(-x) \mathrm{K}(-x, y) d x,
$$

e cambiando $y$ in $-y$ :

$$
f(-y)=\varphi(-y)-\int_{0}^{y} \varphi(x) \mathrm{K}(x,-y) d x-\int_{0}^{y} \varphi(-x) \mathrm{K}(-x,-y) d x .
$$

Poniamo:

$$
\begin{array}{rll}
f(y)=f_{1}(y) \quad, \quad f(-y)=f_{2}(y), \\
\varphi(y)=\varphi_{1}(y) \quad, \quad \varphi(-y)=\varphi_{2}(y), \\
\mathrm{K}(x, y)=-\mathrm{S}_{11}^{(0)}(x, y) \quad, \quad \mathrm{K}(-x, y)=-\mathrm{S}_{12}^{(0)}(x, y), \\
\mathrm{K}(x,-y)=\mathrm{S}_{21}^{(0)}(x, y) \quad, \quad \mathrm{K}(-x,-y)=\mathrm{S}_{22}^{(0)}(x, y),
\end{array}
$$

allora le due equazioni precedenti si scriveranno:

$$
\begin{aligned}
& f_{I}(y)=\varphi_{1}(y)-\int_{0}^{y} \varphi_{1}(x) \mathrm{S}_{11}^{(0)}(x, y) d x-\int_{0}^{y} \varphi_{2}(x) \mathrm{S}_{12}^{(o)}(x, y) d x \\
& f_{2}(y)=\varphi_{2}(y)-\int_{0}^{y} \varphi_{1}(x) \dot{S}_{21}^{(0)}(x, y) d x-\int_{0}^{y} \varphi_{2}(x) \mathrm{S}_{22}^{(0)}(x, y) d x
\end{aligned}
$$

e quindi da queste equazioni potremo ricavare $\varphi_{1}(x)$ e $\varphi_{2}(x)$ definite ambedue nell'intervallo $(\mathrm{o}, a)$ mediante le formule [cfr. Art. I, $\S$ io, formule (19) (20]):

$$
\begin{aligned}
& \varphi_{1}(y)=f_{1}(y)-\int_{0}^{y} f_{1}(x) s_{11}^{(0)}(x, y) d x-\int_{0}^{y} f_{2}(x) s_{12}^{(0)}(x, y) d x \\
& \varphi_{2}(y)=f_{2}(y)-\int_{0}^{y} f_{2}(x) s_{21}^{(0)}(x, y) d x-\int_{0}^{y} f_{2}(x) s_{22}^{(0)}(x, y) d x
\end{aligned}
$$

Definendo la funzione $\mathrm{R}(x, y)$ per $y>x>-y, a>y>-a$, mediante le seguenti relazioni:

$$
\begin{array}{r}
s_{11}^{(0)}(x, y)=-\mathrm{R}(x, y), s_{12}^{(0)}(x, y)=-\mathrm{R}(-x, y), s_{21}^{(0)}(x, y)=\mathrm{R}(x,-y), \\
s_{22}^{(0)}(x, y)=\mathrm{R}(-x,-y) \quad\left(\begin{array}{l}
y>x>0 \\
a>y>0
\end{array}\right),
\end{array}
$$

potremo sostituire alle precedenti equazioni l'unica formula:

$$
\varphi(y)=f(y)+\int_{-y}^{y} f(x) \mathrm{R}(x, y) d x \quad, \quad(a>y>-a),
$$

la quale risolve la equazione funzionale (IO). 
Supponiamo ora di avere la equazione funzionale:

$$
f(y)=\varphi(y)+\int_{\alpha y}^{y} \varphi(x) \mathrm{K}(x, y) d x \quad\left(\begin{array}{l}
0>\alpha>-\mathrm{I} \\
a>y>\alpha a
\end{array}\right),
$$

con $\varphi(x)$ funzione incognita.

Essa potrà ridursi alla (IO), basterà perciò supporre:

$$
\mathrm{K}(x, y)=0 \text { per }\left\{\begin{array} { c } 
{ \alpha y > x > - y } \\
{ a > y > \alpha a }
\end{array} \text { e per } \left\{\begin{array}{l}
\alpha a>y>-a \\
y>x>-y
\end{array}\right.\right.
$$

e prolungare la funzione $f(y)$ per $y$ compreso fra $\alpha a$ e $-a$ in manięra arbitraria pur di conservarla atta alla integrazione e finita.

Ne risulteranno:

$$
\begin{gathered}
\mathrm{S}_{12}^{(0)}=0 \\
\mathrm{~S}_{22}^{(0)}=0 \\
\text { per }\left\{\begin{array}{l}
y>x>-\alpha y \\
a>y>0
\end{array}\right.
\end{gathered}
$$

e per conseguenza $S_{12}^{(i)}, S_{21}^{(i)}, S_{22}^{(i)}$ saranno nulle per quei valori delle variabili $x, y$ pei quali rispettivamente abbiamo indicato esser. nulle $\mathrm{S}_{12}^{(0)}, \mathrm{S}_{2 \mathrm{I}}^{(0)}, \mathrm{S}_{22}^{(0)}$ e quindi per gli stessi valori delle variabili $x, y$ saranno pure nulle $s_{12}^{(0)}, s_{21}^{(0)}, s_{22}^{(0)}(6)$ e perciò finalmente la (I I) si ridurrà a:

$$
\varphi(y)=f(y)+\int_{a y}^{y} f(x) \mathrm{R}(x, y) d x
$$

giacché $\mathrm{R}(x, y)$ al pari di $\mathrm{K}(x, y)$ sarà tale che:

$$
\mathrm{R}(x, y)=0 \quad \operatorname{per}\left\{\begin{array} { c } 
{ \alpha y > x > - y } \\
{ a > y > \alpha a }
\end{array} \text { e per } \left\{\begin{array}{l}
\alpha a>y>-a \\
y>x>-y .
\end{array}\right.\right.
$$

8. Ciò premesso passiamo alla risoluzione della equazione funzionale:

$$
f(y)-f(0)=\int_{\alpha y}^{y} \theta(x) \mathrm{H}(x, y) d x \quad, \quad(a>y>\alpha a),
$$

(6) Questi risultati divengono pressoché intuitivi esaminando la questione da un punto di vista geometrico, ossia costruendo nel piano $x, y$ i campi nei quali sono individuate le diverse funzioni che si considerano, marcando le porzioni di essi ove le funzioni stesse si annullano, ed i cammini delle integrazioni mediante le quali si passa dalle $\mathrm{S}_{r, t}^{(i)}$ alle $\mathrm{S}_{r, t}^{2+\mathrm{r}}$. Noi ci risparmiamo di riportare qui tali figure che il lettore può da sè facilmente disegnare. 
nella ipotesi $0>\alpha>-\mathrm{I}$. Supponendo che $f(y), f^{\prime}(y), \mathrm{H}(x, y), \mathrm{H}_{2}(x, y)$ siano funzioni finite e continue per $x, y$ variabili rispettivamente negl'intervalli $(\alpha y, y),(\alpha a, a)$ e $\theta(x)$ sia la funzione incognita nell'intervallo $(\alpha a, a)$ avremo che le equazioni (4), (5) e (9) seguiteranno a sussistere; e il campo entro il quale si dovranno considerare le funzioni $\mathrm{H}(x, y), \mathrm{H}_{2}(x, y), \mathrm{L}(x, y)$ sarà costituito dalla figura compresa fra le linee $y=a\left(\mathrm{AA}^{\prime}\right), y=\alpha a\left(\mathrm{BB}^{\prime}\right)$, $y=x(\mathrm{AB}), y=\alpha x\left(\mathrm{~B}^{\prime} \mathrm{A}^{\prime}\right)$. Si tirino le rette aventi per equazioni $y=\alpha x$, $y=\alpha^{2} x, y=\alpha^{3} x, \ldots$ Preso quindi un punto M entro la figura si tracci a

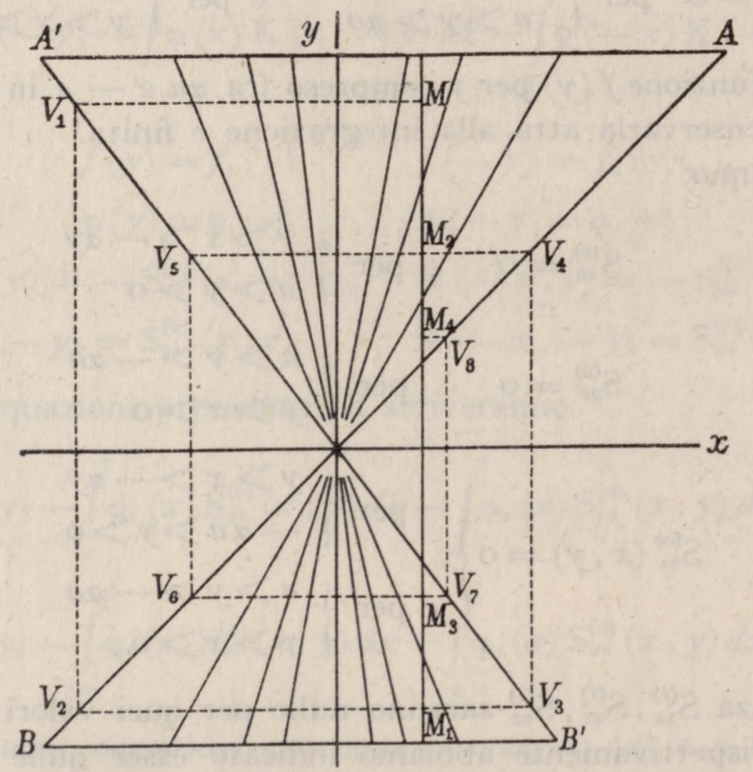

(Nellla figura si è supposto $n=5$ ).

partire da esso una poligonale $\mathrm{MV}_{1} \mathrm{~V}_{2}, \mathrm{~V}_{3} \ldots$ con i lati paralleli agli assi $x, y$ ed avente successivamente $\mathrm{i}$ vertici situati sulle rette $\mathrm{AB}$ e $\mathrm{A}^{\prime} \mathrm{B}^{\prime}$ e si determinino i punti $\mathrm{M}_{\mathrm{o}}=\mathrm{M}, \mathrm{M}_{\mathrm{r}}, \mathrm{M}_{2}, \ldots$, interni alla figura che la poligonale ha a comune colla parallela all'asse $y$ condotta per M. È facile riconoscere che se M è compreso fra le rette $y=\alpha^{n-1} x$ e $y=\alpha^{n+1} x$, i detti punti saranno $n$, e le coordinate del punto $\mathrm{M}_{i}$ saranno $x, \alpha^{i} y$.

Definiamo ora la funzione $\mathrm{K}(x, y)$ prendendone il valore nel punto $\mathrm{M}$ dato da:

$$
\begin{gathered}
\mathrm{L}(x, y)-\alpha \lambda(y) \mathrm{L}(x, \alpha y)+\alpha^{2} \lambda(\alpha y) \lambda(y) \mathrm{L}\left(x, \alpha^{2} y\right)+\cdots \\
\cdots+(-\mathrm{I})^{n} \alpha^{n} \lambda\left(\alpha^{n-1} y\right) \lambda\left(\alpha^{n-2} y\right) \cdots \lambda(y) \mathrm{L}\left(x, \alpha^{n} y\right) \\
=\frac{\mathrm{I}}{\mathrm{H}(y, y)}\left\{\mathrm{H}_{2}(x, y)-\alpha \frac{\mathrm{H}(\alpha y, y)}{\mathrm{H}(\alpha y, \alpha y)} \mathrm{H}_{2}(x, \alpha y)\right. \\
+\alpha_{2} \frac{\mathrm{H}(\alpha y, y)}{\mathrm{H}(\alpha y, \alpha y)} \frac{\mathrm{H}\left(\alpha^{2} y, \alpha y\right)}{\mathrm{H}\left(\alpha^{2} y, \alpha^{2} y\right)} \mathrm{H}_{2}\left(x, \alpha^{2} y\right)-\cdots \\
\left.\cdots+(-\mathrm{I})^{n} \alpha^{n} \frac{\mathrm{H}(\alpha y, y)}{\mathrm{H}(\alpha y, \alpha y)} \frac{\mathrm{H}\left(\alpha^{2} y, \alpha y\right)}{\mathrm{H}\left(\alpha^{2} y, \alpha^{2} y\right)} \cdots \frac{\mathrm{H}\left(\alpha^{n} y, \alpha^{n-x} y\right)}{\mathrm{H}\left(\alpha^{n} y, \alpha^{n} y\right)} \mathrm{H}_{2}\left(x, \alpha^{n} y\right)\right\}
\end{gathered}
$$


vale a dire:

$$
\begin{gathered}
\mathrm{K}(\mathrm{M})=\frac{\mathrm{I}}{\mathrm{H}(y, y)}\left\{\mathrm{H}_{2}\left(\mathrm{M}_{\mathrm{o}}\right)-\alpha \frac{\mathrm{H}\left(\mathrm{V}_{\mathrm{I}}\right)}{\mathrm{H}\left(\mathrm{V}_{2}\right)} \mathrm{H}_{2}\left(\mathrm{M}_{\mathrm{r}}\right)+\alpha^{2} \frac{\mathrm{H}\left(\mathrm{V}_{\mathrm{I}}\right)}{\mathrm{H}\left(\mathrm{V}_{2}\right)} \frac{\mathrm{H}\left(\mathrm{V}_{3}\right)}{\mathrm{H}\left(\mathrm{V}_{4}\right)} \mathrm{H}_{2}\left(\mathrm{M}_{2}\right)-\cdots\right. \\
\left.\cdots+(-\mathrm{I})^{n} \alpha^{n} \frac{\mathrm{H}\left(\mathrm{V}_{\mathrm{I}}\right)}{\mathrm{H}\left(\mathrm{V}_{2}\right)} \frac{\mathrm{H}\left(\mathrm{V}_{3}\right)}{\mathrm{H}\left(\mathrm{V}_{4}\right)} \cdots \frac{\mathrm{H}\left(\mathrm{V}_{2 n-\mathrm{r}}\right)}{\mathrm{H}\left(\mathrm{V}_{2 n}\right)} \mathrm{H}_{2}\left(\mathrm{M}_{n}\right)\right\} .
\end{gathered}
$$

La funzione $\mathrm{K}(x, y)$ sarà in generale discontinua lungo le linee $y=\alpha x$, $y=\alpha^{2} x, y=\alpha^{3} x, \cdots$, e i suoi valori assoluti saranno inferiori ad un numero finito.

Infatti abbiamo:

$$
\frac{H\left(V_{2 s-x}\right)}{H\left(V_{2 s}\right)}=I+\frac{H\left(V_{2 s-x}\right)-H\left(V_{2 s}\right)}{H\left(V_{2 s}\right)} .
$$

Ora la distanza fra i punti $V_{2 s-r} \mathrm{e}_{2 s}$ è inferiore o al più eguale a $\left|a(\mathrm{I}-\alpha) \alpha^{s-\mathrm{x}}\right|$, quindi se $\varepsilon_{s}$ denota il limite superiore dei valori assoluti delle differenze fra i valori di $\mathrm{H}(x, y)$ in tutte le possibili coppie di punti le cui distanze non superano $\left|a(\mathrm{I}-\alpha) \alpha^{s-1}\right|$, avremo:

$$
\left|\mathrm{H}\left(\mathrm{V}_{2 s-1}\right)-\mathrm{H}\left(\mathrm{V}_{2 s}\right)\right| \leq \varepsilon_{s},
$$

e, chiamando $m^{\prime}$ il limite inferiore dei valori assoluti di $\mathrm{H}(x, x)$, sarà:

$$
\left|\frac{\mathrm{H}\left(\mathrm{V}_{2 s-\mathrm{r}}\right)}{\mathrm{H}\left(\mathrm{V}_{2 s}\right)}\right| \leq \mathrm{I}+\frac{\varepsilon_{s}}{m^{\prime}},
$$

d'onde:

$$
\begin{gathered}
|\mathrm{K}(\mathrm{M})| \leq \frac{m^{\prime \prime}}{m^{\prime}}\left\{\mathrm{I}-\alpha\left(\mathrm{I}+\frac{\varepsilon_{1}}{m^{\prime}}\right)+\alpha^{2}\left(\mathrm{I}+\frac{\varepsilon_{1}}{m^{\prime}}\right)\left(\mathrm{I}+\frac{\varepsilon_{2}}{m^{\prime}}\right)-\cdots\right. \\
\left.\cdots+(-\mathrm{I})^{n} \alpha^{n}\left(\mathrm{I}+\frac{\varepsilon_{1}}{m^{\prime}}\right)\left(\mathrm{I}+\frac{\varepsilon_{2}}{m^{\prime}}\right) \cdots\left(\mathrm{I}+\frac{\varepsilon_{n}}{m^{\prime}}\right)\right\}
\end{gathered}
$$

in cui $m^{\prime \prime}$ denota il massimo valore assoluto di $\mathrm{H}_{2}(x, y)$. Ora per la proprietà che hanno le funzioni di esser continue uniformemente abbiamo che:

$$
\lim _{s=0} \varepsilon_{s}=0
$$

quindi la serie indefinita a termini positivi:

$$
\begin{aligned}
& \mathrm{T}=\mathrm{I}-\alpha\left(\mathrm{I}+\frac{\varepsilon_{\mathrm{I}}}{m^{\prime}}\right)+\alpha^{2}\left(\mathrm{I}+\frac{\varepsilon_{\mathrm{I}}}{m^{\prime}}\right)\left(\mathrm{I}+\frac{\varepsilon_{2}}{m^{\prime}}\right)-\cdots \\
& \cdots+(-\mathrm{I})^{n} \alpha^{n}\left(\mathrm{I}+\frac{\varepsilon_{\mathrm{I}}}{m^{\prime}}\right)\left(\mathrm{I}+\frac{\varepsilon_{2}}{m^{\prime}}\right) \cdots\left(\mathrm{I}+\frac{\varepsilon_{n}}{m^{\prime}}\right)+\cdots
\end{aligned}
$$

sarà convergente e per conseguenza:

$$
|\mathrm{K}(\mathrm{M})|<\frac{m^{\prime \prime} \mathrm{T}}{m^{\prime}}
$$

il che prova la proposizione enunciata.

In virtù di quanto abbiamo dimostrato la (9) potrà scriversi dunque:

$$
\psi(y)=\theta(y)+\int_{\alpha y}^{y} \theta(x) \mathrm{K}(x, y) d x
$$


e perciò il procedimento esposto nel $\S 7$ [cfr. formule (I2) e (I3)] ci darà il modo di ricavare la funzione incognita $\theta(x)$ dalla (I4).

9. Noi abbiamo fin qui trattato il caso in cui nella equazione funzionale (3) $\circ\left(3^{\prime}\right)|\alpha|$ fosse minore di $\mathrm{I}$. È evidente che il caso nel quale $|\alpha|>\mathbf{I}$ non presenta difficoltà nuove, giacché si può ricondurre subito al precedente. Basterà perciò cambiare la variabile $y$, prendendo $z=\alpha y$ e avremo:

$$
\int_{\alpha y}^{y} \theta(x) \mathrm{H}(x, y) d x=\int_{z}^{z / \alpha} \theta(x) \mathrm{H}\left(x, \frac{z}{\alpha}\right) d x,
$$

onde, posto $\beta=\mathrm{I} / \alpha$, la equazione funzionale diverrà:

$$
f\left(\frac{z}{\alpha}\right)-f(0)=-\int_{\beta z}^{z} \theta(x) \mathrm{H}\left(x, \frac{z}{\alpha}\right) d x,
$$

in cui $|\beta|<\mathrm{I}$.

Resta il caso in cui $\alpha_{1}=-\mathrm{I}$, nel quale l'equazione funzionale diviene:

$$
f(y)-f(0)=\int_{-y}^{y} \theta(x) \mathrm{H}(x, y) d x \quad, \quad(a>y>-a)
$$

Esso è un caso eccezionale che sfugge alle precedenti considerazioni ed in cui la questione assume un carattere diverso. Intanto è da osservare che in questo caso la $f(y)$ non può più scegliersi arbitrariamente, se si vuole che $\varphi(x)$ risulti finita continua e derivabile, giacché derivando due volte l'equazione precedente si trova:

$$
f^{\prime}(\mathrm{o}) \mathrm{H}_{2}(\mathrm{O}, \mathrm{o})-f^{\prime \prime}(\mathrm{o}) \mathrm{H}(\mathrm{O}, \mathrm{o})=\mathrm{o}
$$

Ma in casi particolari la $f(y)$ può andar soggetta a limitazioni di una natura ancora più restrittiva. Dalla ( $\mathrm{I}_{5}$ ) infatti segue, cambiando $y$ in $-y$ :

$$
f(-y)-f(0)=-\int_{-y}^{y} \theta(x) \mathrm{H}(x,-y) d x,
$$

quindi se, per esempio, $\mathrm{H}(x,-y)=-\mathrm{H}(x, y)$ dovrà essere $f(y)=f(-y)$, mentre dovrà essere $f(y)=-f(-y)$ se $\mathrm{H}(x, y)=\mathrm{H}(x,-y)$ ed il problema funzionale risulterà indeterminato. Noi non staremo ad approfondire qui la questione di risolvere la (I 5), ma ci limiteremo a mostrare a quale classe di questioni essa appartenga.

Si prenda nelle (I 5) e (I6):

$$
\begin{array}{rrr}
f(y)=f_{1}(y) \quad, f(-y)=f_{2}(y) & \quad \theta(x)=\theta_{1}(x) \quad, \quad \theta(-x)=\theta_{2}(x), \\
\mathrm{H}(x, y)=\mathrm{H}_{11}(x, y) & \mathrm{H}(-x, \quad y)=\mathrm{H}_{12}(x, y), \\
-\mathrm{H}(x,-y)=\mathrm{H}_{21}(x, y) \quad, & -\mathrm{H}(-x,-y)=\mathrm{H}_{22}(x, y),
\end{array}
$$


allora esse diverranno [cfr. $§ 7]$ :

$$
\begin{aligned}
& f_{\mathrm{I}}(x)-f_{\mathrm{I}}(\mathrm{0})=\int_{0}^{y}\left[\mathrm{H}_{\mathrm{II}}(x, y) \theta_{\mathrm{I}}(x)+\mathrm{H}_{\mathrm{I} 2}(x, y) \theta_{2}(x)\right] d x, \\
& f_{2}(y)-f_{2}(\mathrm{o})=\int_{0}^{y}\left[\mathrm{H}_{2 \mathrm{I}}(x, y) \theta_{\mathrm{I}}(x)+\mathrm{H}_{22}(x, y) \theta_{2}(x)\right] d x .
\end{aligned}
$$

L'analisi di queste equazioni funzionali è stata fatta nel $\S 5$ della mia prima Nota dell'Accademia dei Lincei, precedentemente citata, quando il determinante:

$$
\left|\begin{array}{l}
\mathrm{H}_{\mathrm{II}}(y, y), \mathrm{H}_{\mathrm{12}}(y, y) \\
\mathrm{H}_{2 \mathrm{I}}(y, y), \mathrm{H}_{22}(y, y)
\end{array}\right| \gtrless 0 .
$$

$\mathrm{Nel}$ nostro caso esso si annulla per $y=0$. Poiché questo determinante compie lo stesso ufficio della funzione $\mathrm{H}(y, y)$ nei problemi considerati nel $\S$ i I dell'Art. I, così si riconosce che la questione si presenta come dello stesso tipo di quella che si incontra allorché $\mathrm{H}(y, y)$ si annulla per $y=0$. [Cfr. l'ultimo teorema del § I I dell'Art. I].

io. Supponiamó che l'equazione funzionale abbia la forma:

$$
f(y)=\int_{p y}^{q y} \varphi(x) \mathrm{H}(x, y) d x,
$$

in cui $|p| q \mid \lessgtr \mathrm{I}$.

Senza alterare la generalità delle nostre considerazioni potremo supporre senz'altro:

Pongasi:

$$
\left|\frac{p}{q}\right|<\mathrm{I} .
$$

avremo:

$$
q y=z,
$$

$$
f\left(\frac{z}{q}\right)=\int_{a s}^{z} \varphi(x) \mathrm{H}\left(x, \frac{z}{q}\right) d x,
$$

essendo $\alpha=p / q$. L'equazione rientra quindi nella classe di equazioni funzionali precedentemente studiate.

Abbiasi ora l'equazione:

$$
f(y)=\int_{p}^{q} \varphi(x, y) \mathrm{K}(x, y) d x,
$$

in cui $p, q$ sono due limiti costanti arbitrarii, escluso il caso $p / q=\mathrm{I}$.

Posto $x y=\xi$, la relazione precedente diverrà:

$$
f(y)=\int_{p y}^{a y} \varphi(\xi) \mathrm{K}\left(\frac{\xi}{y}, y\right) y d \xi
$$


e posto:

$$
\mathrm{K}\left(\frac{\xi}{y}, y\right) y=\mathrm{H}(\xi, y),
$$

l'ultima equazione diverrà:

$$
f(y)=\int_{p y}^{q y} \varphi(\xi) \mathrm{H}(\xi, y) d \xi,
$$

ossia si ridurrà alla (I7). Il problema dunque propostosi da ABEL e di cui fu parlato nel § I dell'Art. I potrà risolversi mediante le fatte considerazioni, supposta $\mathrm{K}(\xi / y, y) y$ finita ed escluso il caso in cui per i limiti si abbia $|p| q \mid=\mathrm{I}$.

ART. III.

I. Dedicheremo questo articolo ad alcune applicazioni delle formule generali esposte nell'Art. I. Il procedimento ivi indicato nei $\S \S$ IO, II dà la soluzione dei vari problemi d'inversione mediante delle operazioni di quadratura; in molti casi esse si eseguiscono con facilità ed in particolare quando le funzioni date sono serie di potenze.

2. Cominciamo dal provare come, eseguendo le dette quadrature, le formule (23) e (24) dell'Art. I conducano subito alla formula di SonINE allorché si suppone che $\mathrm{F}(u)$ sia una serie di potenze.

Prendiamo:

$$
\mathrm{F}(u)=\sum_{0}^{\infty} a_{h} u^{h} \quad, \quad a_{\mathrm{o}}=\mathrm{I},
$$

tenendo presenti le (24) dell'Art. I, avremo:

$$
s_{\circ}(v)=\frac{I}{\Gamma(I-\lambda)} \sum_{0}^{\infty} a_{h+x} \frac{\Gamma(h-\lambda+2)}{\Gamma(h+\mathrm{I})} v^{h},
$$

ovvero, ponendo:

$$
\begin{aligned}
& a_{h+\mathrm{I}} \frac{\Gamma(h-\lambda+2)}{\Gamma(\mathrm{I}-\lambda)}=c_{h+\mathrm{I}}, \\
& s_{\mathrm{o}}(v)=\sum_{0}^{\infty} \frac{c_{h+\mathrm{x}}}{\Gamma(h+\mathrm{I})} v^{h}, \quad c_{\mathrm{o}}=1 .
\end{aligned}
$$

Abbiamo ora:

$$
t_{\mathrm{o}}(v)=\frac{1}{v^{\mathrm{x}-\lambda}}
$$

e perciò:

$$
t_{i}(v)=\frac{v^{i}}{v^{x-\lambda}} \sum_{0}^{\infty} b_{i, s} v^{s} \quad, \quad\left(b_{0}, \mathrm{o}=\mathrm{I}, b_{0}, s=0\right),
$$


onde:

$$
t_{i+\mathrm{I}}(v)=\frac{v^{i+\mathrm{x}}}{v^{\mathrm{I}-\lambda}} \sum_{0}^{\infty} v^{r} \sum_{0}^{r} c_{h+\mathrm{I}} b_{i, r-h} \frac{\Gamma(i+r-h+\lambda)}{\Gamma(i+r+\lambda+\mathrm{I})},
$$

ossia, ponendo:

$$
b_{i, s}=\frac{\beta_{i, s}}{\Gamma(i+s+\lambda)},
$$

si ha la formula ricorrente:

$$
\beta_{i+\mathrm{x}, r}=\sum_{0}^{r} c_{h+\mathrm{x}} \beta_{i, r-h} \quad, \quad\left[\beta_{\mathrm{o}, \mathrm{o}}=\Gamma(\lambda)\right] .
$$

Abbiamo dunque il modo di calcolare tutte le $t_{i}(v)$ e per conseguenza $\Omega(v)$.

3. Osserviamo che supponendo la variabile $v$ complessa e $|v|$ minore del raggio di convergenza della serie (I), la funzione:

$$
\Theta(v)=v^{\mathrm{x}}-\lambda \Omega(v)=\sum_{0}^{\infty}(-\mathrm{I})^{i} v^{\mathrm{x}} \psi^{-\lambda} t_{i}(v),
$$

in virtù delle legge con cui decrescono i limiti superiori dei moduli di $t_{i}(v)$ è una funzione uniforme ed olomorfa. Essa è dunque una serie di potenze di a convergente entro il cerchio di convergenza della (I), quindi risulterà:

$$
\Theta(v)=\sum_{0}^{\infty} e_{k} v^{k}=\sum_{0}^{\infty} v^{k} \sum_{0}^{k}(-\mathrm{I})^{s} b_{s, k-s}
$$

da cui segue:

$$
e_{k}=\sum_{0}^{k}(-\mathrm{I})^{s} b_{s, k-s}=\frac{\mathrm{I}}{\Gamma(k+\lambda)} \sum_{0}^{k}(-\mathrm{I})^{s} \beta_{s, k-s} .
$$

Si ponga:

$$
\varepsilon_{k}=\sum_{0}^{k}(-\mathrm{I})^{s} \beta_{s, k-s} \quad, \quad \varepsilon_{0}=\Gamma(\lambda),
$$

sarà per la (2):

$$
\begin{gathered}
\varepsilon_{k}=\sum_{\mathrm{I}}^{k}(-\mathrm{I})^{s} \sum_{0}^{k} \sum_{h}^{s} c_{h+\mathrm{I}} \beta_{s-\mathrm{I}, k-s-h}=-\sum_{0}^{k} \sum_{h}^{\mathrm{I}} c_{h+\mathrm{I}} \sum_{0}^{k-h-\mathrm{I}}(-\mathrm{I})^{\sigma} \beta_{\sigma, k-h-\sigma-\mathrm{I}} \\
=-\sum_{0}^{k-1} c_{h+\mathrm{I}} \varepsilon_{k-h-\mathrm{I}}=-\sum_{\mathrm{I}}^{k} c_{h} \varepsilon_{k-h} .
\end{gathered}
$$

Riassumendo si hanno le formule:

$$
\begin{gathered}
\mathrm{F}(u)=\sum_{0}^{\infty} a_{h} u^{h} \quad, \quad a_{\mathrm{o}}=\mathrm{I}, \\
c_{h}=\frac{a_{h} \Gamma(h-\lambda+\mathrm{I})}{\Gamma(\mathrm{I}-\lambda)},
\end{gathered}
$$




$$
\begin{gathered}
\varepsilon_{k}=-\sum_{\mathrm{I}}^{k} c_{h} \varepsilon_{k-h}, \\
e_{k}=\frac{\mathrm{I}}{\Gamma(k+\lambda)} \varepsilon_{k}, \\
\frac{\operatorname{sen} \lambda \pi}{\pi} \Theta(u)=\frac{\operatorname{sen} \lambda \pi}{\pi} \sum_{0}^{\infty} e_{k} v^{k}, \\
\Omega(v)=\frac{\Theta(v)}{v^{I-\lambda}},
\end{gathered}
$$

le quali ci permettono di calcolare $\Theta$ e quindi $\Omega$ conoscendo F.

Osserviamo ora che dalla terza delle precedenti equazioni segue:

$$
\sum_{0}^{k} c_{h} \varepsilon_{k-h}=0, \quad(k=\mathrm{I}, 2, \cdots),
$$

mentre:

$$
c_{0} \varepsilon_{0}=\Gamma(\lambda)
$$

perciò se poniamo:

$$
\mathrm{I}+c_{1} x+c_{2} x^{2}+\cdots=\rho(x),
$$

$$
\frac{\mathrm{I}}{\Gamma(\lambda)}\left(\varepsilon_{\mathrm{o}}+\varepsilon_{1} x+\varepsilon_{2} x^{2}+\cdots\right)=\mathrm{I}+\partial_{1} x+\partial_{2} x^{2}+\cdots=\psi(x),
$$

si avrà:

$$
\rho(x) \psi(x)=\mathrm{I},
$$

e quindi:

$$
\left\{\begin{array}{l}
\mathrm{F}(u)=\Gamma(\mathrm{I}-\lambda) \sum_{0}^{\infty} \frac{c_{h}}{\Gamma(h+\mathrm{I}-\lambda)} u^{h} \\
\frac{\operatorname{sen} \lambda \pi}{\pi} \Theta(u)=\frac{\mathrm{I}}{\Gamma(\mathrm{I}-\lambda)} \sum_{0}^{\infty} \frac{\partial_{k}}{\Gamma(k+\lambda)} v^{k},
\end{array}\right.
$$

le quali appunto conducono alla formula di Sonine (cfr. Art. I, § 8).

Il risultato varrebbe anche se la (3) non fosse convergente o si annullasse, ma allora dovremmo attribuire alla (3), (4) e (5) un significato simbolico. La reciprocità delle due serie (6) prova che esse hanno lo stesso cerchio di convergenza.

4. Il precedente risultato può ottenersi anche molto semplicemente dalla equazione (2) colle considerazioni seguenti.

La (2) prova che se poniamo:

$$
\rho(v)=\sum_{0}^{\infty} c_{i} v^{i},
$$


sarà, almeno simbolicamente:

$$
\sum_{0}^{\infty} \beta_{i+\mathrm{I}, r} v^{i+\mathrm{I}+r}=\left(\sum_{0}^{\infty} \beta_{i, r} v^{i+r}\right)\left(\sum_{\mathrm{I}}^{\infty} c_{h} v^{h}\right)=\left(\sum_{0}^{\infty} \beta_{i, r} v^{i+r}\right)[\mathrm{p}(v)-\mathrm{I}],
$$

e perciò:

$$
\beta_{\mathrm{o}, \mathrm{o}}[-\mathrm{I}+\mathrm{p}(v)]^{i}=\sum_{0}^{\infty} \beta_{i, s} v^{i+s},
$$

quindi per avere $v^{x-\lambda} t_{i}(v)$ basterà dividere per $\Gamma(h+\lambda)$ il coefficiente del termine di grado $h(h=\mathrm{I}, 2,3, \cdots, \infty)$ nella serie:

$$
\beta_{0,0}[-I+\rho(v)]^{i} \text {. }
$$

Se dunque scriviamo:

$$
\Omega(v)=\frac{\Theta(v)}{v^{x-\lambda}}
$$

$\Theta(v)$ si otterrà calcolando:

$$
\beta_{0,0}\left[\sum_{0}^{\infty}(-\mathrm{I})^{i}(-\mathrm{I}+\rho(v))^{i}\right]=\theta(v),
$$

e dividendo quindi il coefficiente del termine di grado $h(h=1,2, \cdots, \infty)$ per $\Gamma(h+\lambda)$.

Ora dalla equazione precedente si deduce, almeno simbolicamente:

$$
\theta(v)=\frac{\beta_{o, \circ}}{\rho(v)}
$$

per conseguenza:

$$
\frac{\operatorname{sen} \lambda \pi}{\pi} \theta(v)=\frac{1}{\Gamma(I-\lambda)} \frac{I}{\rho(v)},
$$

da cui seguono immediatamente le formule (6).

5. Esaminiamo ora le formule (2I) e (22) dell'Art. I, supponendo che F (z) sia una serie di potenze.

Poniamo:

$$
\mathrm{F}(z)=\mathrm{I}+\sum_{\mathrm{I}}^{\infty} a_{i} z^{i}
$$

e scriviamo:

$$
s_{i}(z)=z^{i} \sum_{0}^{\infty} c_{i, h} z^{h}
$$

Avremo intanto:

$$
s_{\circ}(z)=\mathrm{F}^{\prime}(z)=\sum_{I}^{\infty} h a_{h} z^{h-1}
$$


quindi:

$$
c_{\mathrm{o}, h}=(h+\mathrm{I}) a_{h+\mathrm{x}} .
$$

Ma:

$$
\begin{gathered}
s_{i+1}(z)=\int_{0}^{z} s_{0}(z-u) s_{i}(u) d u=\int_{0}^{z} u^{i} \sum_{0}^{\infty} \sum_{0}^{r} c_{0}, h c_{i, r-h} u^{r-h}(z-u)^{h} d u \\
=\sum_{0}^{\infty} \sum_{0}^{r} c_{0, h} c_{i, r-h} z^{i+r+1} \int_{0}^{1} \xi^{i+r-h}(\mathrm{I}-\xi)^{h} d \xi \\
=\sum_{0}^{\infty} z^{i+r+1} \sum_{0}^{r} c_{0, h} c_{i, r-h} \frac{\Gamma(h+\mathrm{I}) \Gamma(i+r-h+\mathrm{I})}{\Gamma(i+r+2)}
\end{gathered}
$$

e per conseguenza:

$$
c_{i+\mathrm{I}, r}=\sum_{0}^{r} \frac{c_{\mathrm{o}, h} \Gamma(h+\mathrm{I}) c_{i, r-h} \Gamma(i+r-h+\mathrm{I})}{\Gamma(i+r+2)} .
$$

Posto:

$$
e_{i, r}=c_{i, r} \Gamma(i+r+\mathrm{I}) \text {, }
$$

avremo dunque:

$$
e_{i+\mathrm{x}, r}=\sum_{0}^{r} e_{i, r-h} e_{\mathrm{o}, h}
$$

da cui segue:

$$
\sum_{0}^{\infty} e_{i+1, r} u^{r}=\left(\sum_{0}^{\infty} e_{i, r} u^{r}\right)\left(\sum_{0}^{\infty} e_{0, r} u^{r}\right),
$$

e perciò:

$$
\sum_{0}^{\infty} e_{i, r} u^{r}=\left(\sum_{0}^{\infty} e_{0, r} u^{r}\right)^{i+r}
$$

Osserviamo che se anche la serie $\sum_{0}^{r} e_{0, r} u^{r}$ non fosse convergente, le due formule precedenti conserverebbero il loro significato simbolico e lo stesso può dirsi delle formule seguenti.

Per ottenere dunque $s_{i}(z)$ basterà fare la potenza $(i+1)^{\text {esima }}$ della serie $\sum_{0}^{\infty} e_{0, r} z^{r}$, quindi moltiplicarla per $z^{i}$ e dividere per $\Gamma(i+r+1)$ il coefficiente del termine di grado $i+r(r=0, \mathrm{I}, 2, \cdots, \infty)$.

Si noti ora, come già facemmo nel caso precedentemente studiato, che supponendo $z$ complessa, finché $|z|$ è minore del raggio del circolo di convergenza della serie (7) la funzione:

$$
\Theta(z)=\sum_{0}^{\infty}(-\mathrm{I})^{i} s_{i}(z),
$$

sarà olomorfa; essa sarà dunque esprimibile con una serie di potenze di $z$ convergente entro il cerchio di convergenza della (7). 
Per ottenerla si formi:

$$
\sum_{0}^{\infty}(-\mathrm{I})^{i} z^{i}\left(\sum_{0}^{\infty} e_{0, r} z^{r}\right)^{i+\mathrm{I}}=\frac{\sum_{0}^{\infty} e_{0, r} z^{r}}{\mathrm{I}+z \sum_{0}^{\infty} e_{r} e_{0}, z^{r}}=\sum_{0}^{\infty} b_{h} z^{h}
$$

avremo allora:

$$
\Theta(z)=\sum_{0}^{\infty} \frac{b_{h}}{\Gamma(h+\mathrm{I})} z^{h}
$$

Dalle (7), (8) e (9) si deduce, scrivendo per semplicità $e_{h}$ invece di $e_{0, h}$ :

$$
\mathrm{F}(z)=\mathrm{I}+\sum_{0}^{\infty} \frac{e_{h}}{\Gamma(h+2)} z^{h+1},
$$

onde potremo enunciare la proposizione seguente:

Posto:

$$
\begin{gathered}
\sigma(z)=\sum_{0}^{\infty} e_{h} z^{h}, \\
p(z)=\frac{\sigma(z)}{1+z \sigma(z)}=\sum_{0}^{\infty} b_{h} z^{h},
\end{gathered}
$$

e quindi:

$$
\begin{gathered}
\mathrm{F}(z)=\mathrm{I}+\sum_{0}^{\infty} \frac{e_{h}}{\Gamma(h+2)} z^{h+1}, \\
\Theta(z)=\sum_{0}^{\infty} \frac{b_{h}}{\Gamma(h+\mathrm{I})} z^{h},
\end{gathered}
$$

si avrà che l'integrale:

$$
f(y)-f(\alpha)=\int_{\alpha}^{y} \varphi(x) \mathrm{F}[\lambda(x)-\lambda(y)] d x,
$$

si invertirà mediante la formula:

$$
\varphi(y)=f^{\prime}(y)+\lambda^{\prime}(y) \int_{\alpha}^{y} f(x) \Theta[\lambda(x)-\lambda(y)] d x,
$$

e per la validità delle formule precedenti basterà mantenersi entro il cerchio di convergenza della serie (I4), anche se le (12) e (I3) non fossero convergenti, attribuendo in questo caso a queste ultime un significato simbolico.

6. Le formule precedenti possono verificarsi anche direttamente col procedimento seguente.

Affinché:

$$
\varphi(y)=f^{\prime}(y)+\lambda^{\prime}(y) \int_{\alpha}^{y} f^{\prime}(x) \Theta[\lambda(x)-\lambda(y)] d x,
$$


verifichi l'equazione funzionale:

$$
f(y)-f(\alpha)=\int_{\alpha}^{y} \varphi(x) \mathrm{F}[\lambda(x)-\lambda(y)] d x \quad, \quad \mathrm{~F}(0)=\mathrm{I},
$$

dovremo avere:

$$
\begin{gathered}
f(y)-f(\alpha)=\int_{\alpha}^{y} f^{\prime}(x) \mathrm{F}[\lambda(x)-\lambda(y)] d x \\
+\int_{\alpha}^{y} \lambda^{\prime}(x) \mathrm{F}[\lambda(x)-\lambda(y)] \int_{\alpha}^{x} f^{\prime}(\xi) \Theta[\lambda(\xi)-\lambda(x)] d \xi \\
=\int_{\alpha}^{y} f^{\prime}(x) \mathrm{F}[\lambda(x)-\lambda(y)] d x \\
+\int_{\alpha}^{y} f^{\prime}(\xi) d \xi \int_{\xi}^{y} \mathrm{~F}[\lambda(x)-\lambda(y)] \Theta[\lambda(\xi)-\lambda(x)] \lambda^{\prime}(x) d x,
\end{gathered}
$$

quindi:

$$
\int_{\xi}^{y} \mathrm{~F}[\lambda(x)-\lambda(y)] \Theta[\lambda(\xi)-\lambda(x)] \lambda^{\prime}(x) d x=\mathrm{I}-\mathrm{F}[\lambda(\xi)-\lambda(y)],
$$

ovvero:

$$
-\int_{0}^{z} \mathrm{~F}(u) \Theta(z-u) d u=\mathrm{I}-\mathrm{F}(z)
$$

che può anche scriversi:

$$
\mathrm{F}(z)-\mathrm{F}(0)=\int_{0}^{z} \mathrm{~F}(u) \Theta(z-u) d u
$$

Posto:

$$
\begin{aligned}
& \mathrm{F}(u)=\sum_{0}^{\infty} a_{h} u^{h}, \\
& \Theta(u)=\sum_{0}^{\infty} \partial_{h} u^{h},
\end{aligned}
$$

dall'equazione precedente risulterà:

$$
\sum_{0}^{\infty} z^{r+x} \sum_{0}^{r} a_{h} \partial_{r-h} \int_{0}^{I} v^{h}(\mathrm{I}-v)^{r-h} d v=\sum_{0}^{\infty} a_{r+x} z^{r+1}
$$

onde:

$$
a_{r+1}=\sum_{0}^{r} a_{h} a_{r-h} \int_{0}^{x} v^{h}(\mathrm{I}-v)^{r-h} d v=\sum_{0}^{r} \frac{a_{h} \Gamma(h+1) \partial_{r-h} \Gamma(r-h+1)}{\Gamma(r+2)} .
$$


Scriviamo:

$$
\begin{gathered}
a_{h+1} \Gamma(h+2)=e_{h}=g_{h+1}, \\
\partial_{h} \Gamma(h+1)=b_{h},
\end{gathered}
$$

otterremo:

$$
e_{r}=\sum_{0}^{r} g_{h} b_{r-h}, \quad(r=0,1,2, \cdots),
$$

equazione che equivale, almeno simbolicamente, all'altra:

$$
\sum_{0}^{\infty} e_{r} z^{r}=\left(\sum_{0}^{\infty} b_{r} z^{r}\right)\left(\sum_{0}^{\infty} g_{r} z^{r}\right)
$$

Ma:

$$
\sum_{0}^{\infty} g_{r} z^{r}=\mathrm{I}+z \sum_{0}^{\infty} e_{r} z^{r}
$$

quindi:

$$
\sum_{0}^{\infty} e_{r} z^{r}=\left(\sum_{0}^{\infty} b_{r} z^{r}\right)\left(1+z \sum_{0}^{\infty} e_{r} z^{r}\right),
$$

onde si avranno immediatamente le (I3), (I4) e (I5).

Gli esempi della stessa specie di quelli ora considerati potrebbero moltiplicarsi all'infinito, noi non staremo a trattarne altri, non presentando essi che difficoltà puramente di calcolo ${ }^{(7)}$.

(7) A complemento delle notizie contenute nell'Art. I, debbo aggiungere che durante la stampa del presente scritto il sig. LE Roux ha pubblicato nel fascicolo del i 8 gennaio dei "Comptes rendus de l'Académie des Sciences" una Nota: Sur l'équation des télégraphistes, nella quale accenna ad un teorema sulle funzioni ricavate mediante integrali definiti, rimandando alla sua tesi di laurea, lavoro però che non ho potuto ancora procurarmi. 